\title{
DETERMINATION of STEPSIZE PARAMETERS \\ for \\ INTERMOLECULAR VIBRATIONAL ENERGY TRANSFER
}

Final Report

for Period May 1, 1987 - December 31, 1991

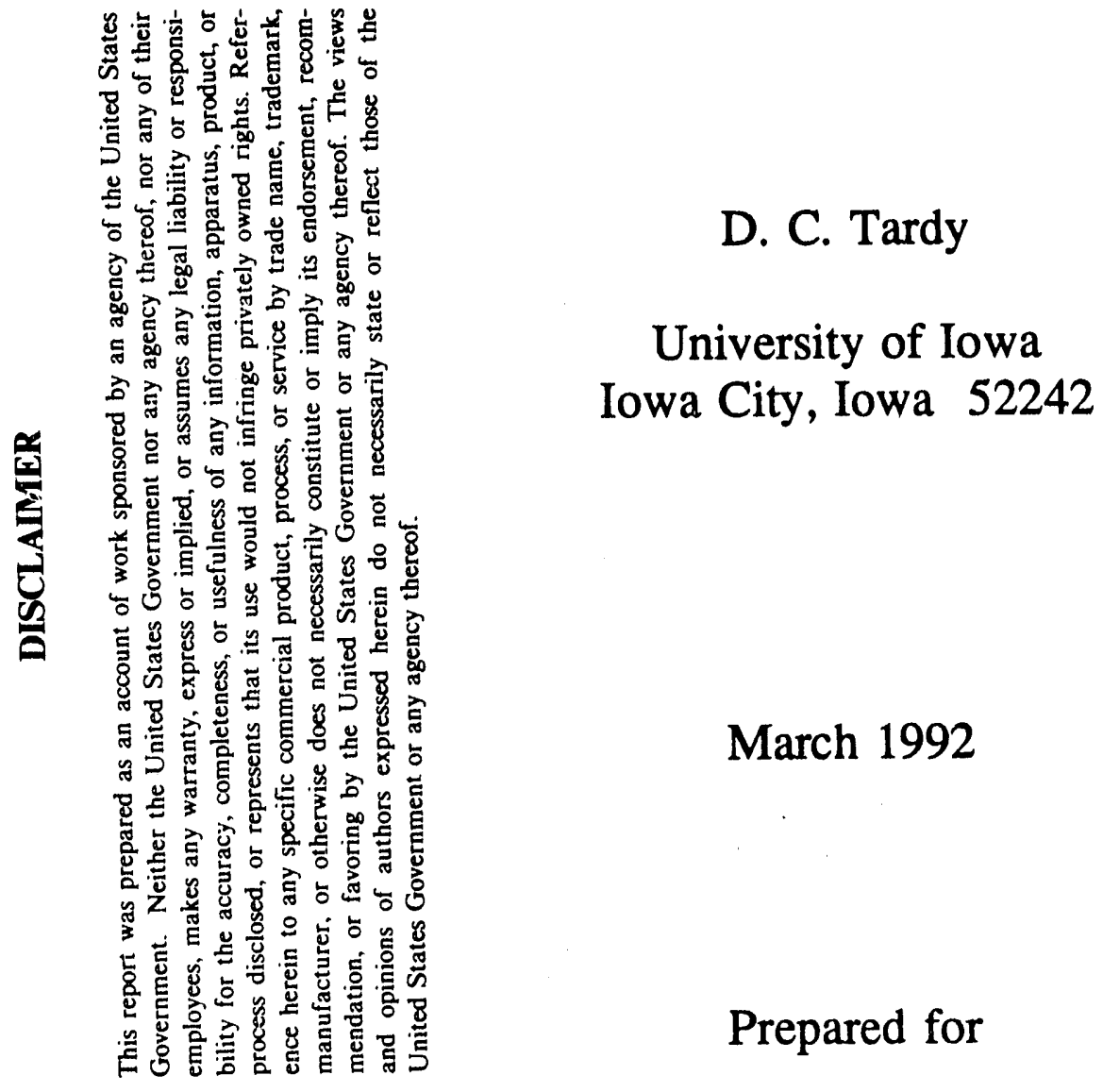

THE U. S. DEPARTMENT OF ENERGY AGREEMENT NO. DE-FG02-87ER 13700 


\section{NOTICE}

This report was prepared as an account of work sponsored by the United States Government. Neither the United States nor the Department of Energy, nor any of their empolyees, nor any of their contractors, subcontractors, or their employees, makes any warranty, express or implied, or assumes any legal liability or responsibility for the accuracy, completeness, or usefulness of any information, apparatus, product or process disclosed or represents that its use would not infringe privately-owned right. 


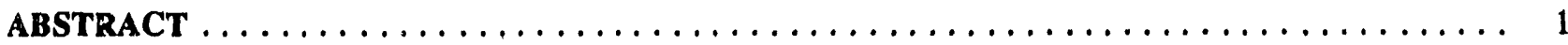

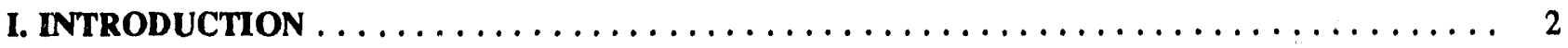

II. ENERGY TRANSFER EXPERIMENTS and CALCULATIONS $\ldots \ldots \ldots \ldots \ldots \ldots \ldots \ldots \ldots$

Experiments Probing the Acoustic Wave $\ldots \ldots \ldots \ldots \ldots \ldots \ldots \ldots \ldots \ldots \ldots \ldots \ldots$

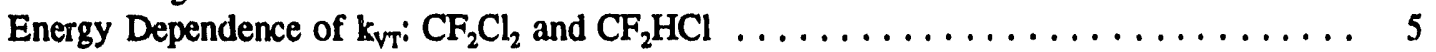

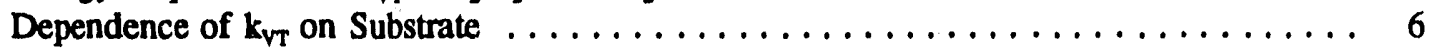

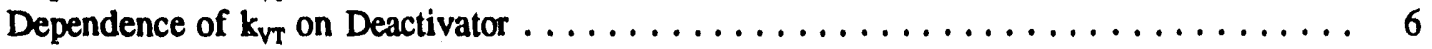

A Simple Model for $k_{\mathrm{VT}}$ for Polyatomics $\ldots \ldots \ldots \ldots \ldots \ldots \ldots \ldots \ldots \ldots$

Experiments Probing the Gas Density: Heterodyne Experiments $\ldots \ldots \ldots \ldots \ldots \ldots \ldots \ldots$

Relating the Difference in Optical Path to Changes in the Gas

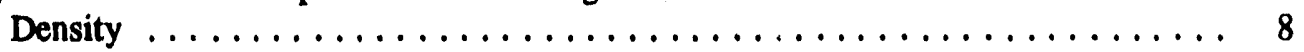

Experiments Probing the Infrared Emission $\ldots \ldots \ldots \ldots \ldots \ldots \ldots \ldots \ldots \ldots \ldots \ldots$

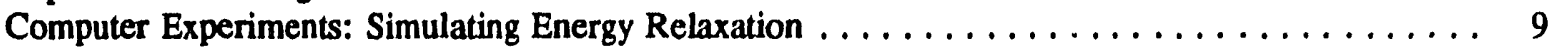

III. HIGH TEMPERATURE COMPLICATIONS fOr UNIMOLECULAR REACTIONS $\ldots \ldots \ldots \ldots 12$

High Temperature Deviations from the Approximate RRKM Expression $\ldots \ldots \ldots \ldots \ldots \ldots \ldots 12$

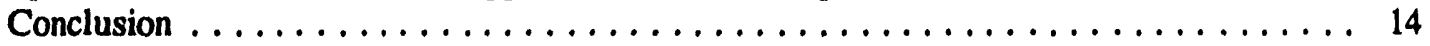

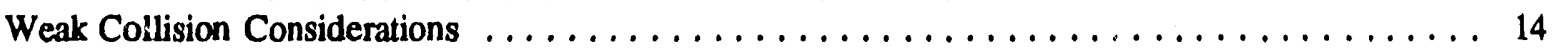

Calculation of Vibrational-Energy Transfer Quantities at High

Temperatures ............................. 16

Energy level Specification for the Collision Process $\ldots \ldots \ldots \ldots \ldots \ldots \ldots \ldots$

IV. REACTIONS STUDIED in which ENERGY TRANSFER may be IMPORTANT $\ldots \ldots \ldots \ldots 18$

Perfluorcyclopropene: production of tetrafluoroallene and

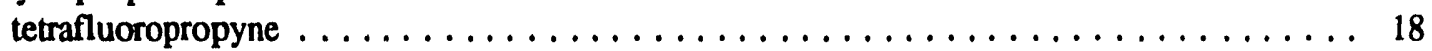

1,2-Difluorodiiodoethene: Production of difluoroacetylene $\ldots \ldots \ldots \ldots \ldots \ldots \ldots \ldots \ldots$

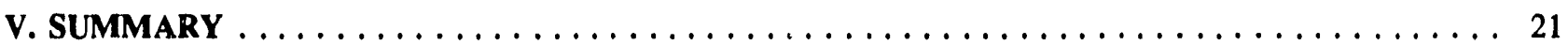

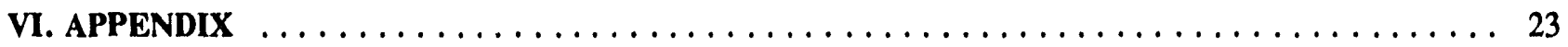

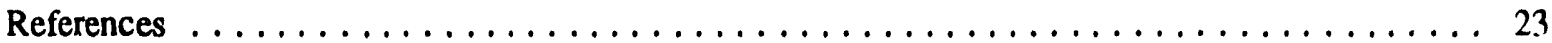

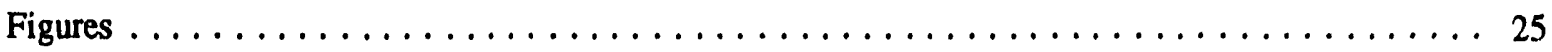

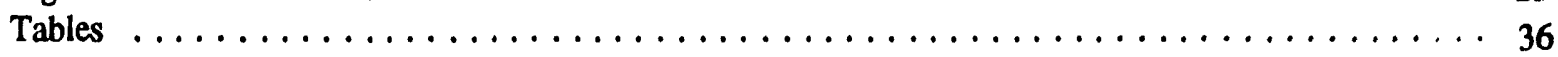

\section{ABSTRACT}

Intermolecular energy transfer of highly excited polyatomic molecules plays an important role in many complex chemical systems: combustion, high temperature and atmospheric chemistry. By monitoring the relaxation of internal energy we have observed trends in the collisional efficiency $(\beta)$ for energy transfer as a function of the substrate's excitation energy and the complexities of substrate and deactivator. For a given substrate $\beta$ increases as the deactivator's mass increases to $\sim 30$ amu and then exhibits a nearly constant value; this is due to a mass mismatch between the atoms of the colliders. In a homologous series of substrate moiecules $\left(C_{3}-C_{8}\right) \beta$ decreases as the number of atoms in the substrate increases; replacing $\mathrm{F}$ with $\mathrm{H}$ increases $\beta$. All substrates, except for $\mathrm{CF}_{2} \mathrm{Cl}_{2}$ and $\mathrm{CF}_{2} \mathrm{HCl}$ below $10,000 \mathrm{~cm}^{-1}$, exhibited that $\beta$ is independent of energy, i.e. $\langle\Delta \mathrm{E}\rangle_{\text {dll }}$ is linear with energy. The results are interpreted with a simple model which considers that $\beta$ is a function of the oscillators energy and its vibrational frequency. Limitations of current approximations used in high temperature unimolecular reactions were evaluated and better approximations were developed. The importance of energy transfer in product yields was observed for the photoactivation of perfluorocyclopropene and the photoproduction of difluoroethyne. 


\section{INTRODUCTION}

Understanding chemical processes from a kinetics and thermodynamics point of view will provide the necessary information so that reaction product yields can be optimized with a concomitant decrease in the production $C I$. undesirable secondary products. The direct benefits of this understanding will be both economical and environmental; products can be produced less expensively without contaminating the environment with unacceptable and/or toxic waste.

The phenomenological (macroscopic) conversion of reactants $(R)$ to products $(P)$ can be broken down into a series of simpler chemical (mesoscopic) steps involving intermediates; each of these steps can be further broken down. As more understanding is required more detailed steps are specified; until ultimately a microscopic description of the reactants and products (energy states, geometry, etc) with a description of the collision (impact parameters, molecular orientation) is necessary. Energy transfer ${ }^{l}$ is an important component to these steps and is especially critical for the understanding of high temperature systems, combustion and atmospheric chemistry.

At the microscopic level, fundamental input includes: the specification of energy into the various degrees of motion, the angular momentum of reactants and products, molecular orientation, impact parameters and the interaction energy. The cross section for a particular step (the fundamental quantity of interest) relates to the probability of the specified reactant forming the product in its specified state. The cross section can be appropriately averaged to obtain the desired macroscopic rate coefficient. These procedures are well defined; however, various models are assumed to simplify the calculation. Such acceptable assumptions include statistical energy distributions and random lifetimes for unimolecular reactions.

The macroscopic description requires fewer variables: pressure, temperature, substrate, deactivator. Phenomenologically, the rate for the process of interest is determined as a function of these variables. A problem results in trying to relate these experimental variables directly to the microscopic parameters; there is not a unique one for one relationship. For example, the pressure relates to the gas density at a given temperature which also relates to the collision number via the collision cross section (which is also temperature dependent). For systems in thermal equilibrium the temperature determines the total energy; this energy is partitioned into the various modes: translation (relative velocity), rotation, vibration, etc.

For simplicity the rate coefficient is often artificially factored into apparently independent quantities. An example is the description of the falloff for a unimolecular reaction in terms of the collision frequency and an efficiency factor, the efficiency is in turn related to an average energy transferred. Quite frequently this factoring is strictly pragmatic; its advantage is that it is simple, even though it may not be physically realistic. The ultimate goal of this factoring is to be able to predict behavior for unknown systems or for known systerns with unaccessible experimental conditions by applying correction factors to the standard systems.

The dependence of experimental parameters can often be further scrambled by the experimental methodology which is used. It is extremely important that differentiation of preparation, relaxation and observation times are made. Specifically, to simplify the data processing for a pulsed experiment it is important that the preparation of the reactant take place on a time scale short compared to the process being monitored; the observation window should also be short compared to the relaxation. If these conditions are not satisfied, then proper deconvolution must be implemented. Normally, the response functions are not known so approximations are used and potential unknown errors are introduced. State to state experiments are preferable; but polyatomics which have a high density of energy states make these experiments exceedingly difficult. Factors which often determine the experimental methodology are cost, time and how the data will be used.

The macroscopic results and the microscopic description are related: synthesis is building up the macroscopic description from the microscopic steps while analysis is the reverse procedure. It is hoped that apriori quantum calculations and statistical mechanics will be able to describe all chemical processes so that synthesis is the procedure used. However, with present computers (hardware and software) this hope has only been realized for small systems: 5 atoms or less. At this time, a viable alternate is to model the microscopic behavior with a few parameters and then to calibrate these parameters with known reactions. The level of parameterization depends on the objective: whether one is interested in describing the behavior or to understand the behavior. Parameters can be found to mimic observed behavior but only become relevant when they can predict behavior and provide fundamental understanding. However, when simplified or abbreviated or physically unrealistic models are used it is important that the limitations of the model are known.

The fundamental steps in the overall reaction often involve intermolecular cnergy transfer, systems that 
specifically depend on intermolecular energy transfer are: laser induced chemistry, pyrolysis, combustion and atmospheric studies at high and low temperatures and pressures. Modeling of such complicated systems requires rate coefficients at various temperatures and pressures; which depend on the temperature dependence of energy transfer. Thus, energy transfer information obtained in slate to state or bulk experiments are necessary in charting out energy evolution maps in complex systems.

The work described in this report relates to the importance of collisional energy transfer, i.e. the climbing and descending the energy ladder via bimolecular gas phase collisions for chemical processes. We have studied some of the parameters which influence energy transfer via experiments and computer calculations; developed experimental methodologies and analysis schemes; determined the effect of energy transfer on high temperature unimolecular systems and assessed the limitations of commonly used approximations; and we have observed new chemical reactions in which energy transfer exhibited specific behavior. 


\section{ENERGY TRANSFER EXPERIMENTS and CALCULATIONS}

In general, kinetic experiments measure a rate of transport, e.g. in the case of a unimolecular reaction in the low pressure (second order) region, the rate of reaction of product formation is related to the transport of molecules from nonreactive energy levels to reactive levels. Fundamentally, this involves an energy flow (energy/time), which is observed as the rate of formation of products. The rate coefficient for this flow has pragmatically been divided into two terms: the collision rate and the probability of transfer per collision. It can be seen that for a given rate coefficient a variety of collision rates and probabilities are possible: e.g. a high collision rate with a low probability for transfer or a low collision rate with a high probability for transfer. Both will meet the requirement for a given rate coefficient. When factoring an observation into factors it is important that all factors are given. Thus, it is important that both collision rate and probability are specified.

The observed collision or reaction rate coefficient is highly dependent upon the particulir experiment: the distribution of initially prepared states of the reactants and the final states which are detecte. The phenomenological rate coefficient is obtained by a suitable average of the cross section for energy transfer. The detailed cross section is not only an indicator of the efficiency for a given product but also a measure for the energy disposition, i.e. whether vibrational energy of the substrate $(A)$ is transferred to relative translation (V-T transfer), to rotation (V-R) or to vibration (V-V) of the deactivator (M). The relevance of energy disposal is determined by the specific experiment. Specific experiments will provide information on the following step:

$$
A(E)+M\left(E_{2}\right) \rightarrow A\left(E^{\prime}\right)+M\left(E_{2}^{\prime}\right)
$$

where the primes designate the final energies and subscript 2 corresponds to the deactivator $(\mathrm{M})$. State to state experiments, i.e. observing the outcome of single collisions, will provide detailed probailities for the amounts of vibrational energy transferred to each potential energy sink. As the experiment loses resolution (temporal, spatial and energy) more averaging is encountered and the details are lost. Attempts to obtain the microscopic details are made by modeling the results; the interpretation may not be unique for a given set of observations.

These complications are illustrated in Figure 1 for the simulated relaxation of cyclohexene by a $300 \mathrm{~K}$ deactivator with an exponential model which removes an average of $500 \mathrm{~cm}^{-1}$ per substrate collision. The populations are plotted as a function of number of collisions (time) and vibrational energy; the average energies, $\langle E\rangle$ (solid line) and $\left\langle E^{2}\right\rangle^{0.5}$ (dashed line) are also displayed. Three initial population distributions are shown (plots A, B and C): A) a delta function with input at $25000 \mathrm{~cm}^{-1}$, a box energy function between 22000 and $28000 \mathrm{~cm}^{-1}$ and a Boltzmann distribution at $\left.1500 \mathrm{~K}(\angle E\rangle=22000 \mathrm{~cm}^{-1}\right)$. As the number of initially populated energy levels increases (progressing from $A$ to $B$ to $C$ ), the origin of the levels generating a specific $E^{\prime}$ becomes fuzzy and detailed interpretaion becomes susceptible to error. The most detailed information is obtained for observations close to the initial distribution. Various average quantities (such as moments of the energy), which exhibit different relaxation functions can be used to unravel some of the details, i.e. the small but measurable differences between $\langle E\rangle_{1}$ and $\left\langle E^{2}\right\rangle^{0.5}$ are illustrated in plots $A, B$ and $C$. These average quantities can be measured directly by monitoring the substrate or indirectly by monitoring the deactivator. The latter involves further modeling but can provide unique information.

We have used a pulsed $\mathrm{CO}_{2}$ laser (photon encrgy $\sim 1000 \mathrm{~cm}^{-1}$ ) as the excitation source: for our experiments the preparation time can be considered short relative to the relaxation time so that deconvolution of these times is not necessary. However, although the initial $\langle\mathrm{E}\rangle$ is known, the detailed population distribution function resulting from the multiple photon excitation is an unknown parameter; a Boltzmann population with the same $\langle E\rangle$ has been reported. Except for the $\mathrm{C}_{2} \mathrm{H}_{4}$ heterodyne experiments described below, the $\mathrm{CO}_{2}$ photon is absorbed by the C-F stretching vibrational mode; rapid intramolecular vibrational energy relaxation is assumed at high levels of excitation. A potential problem with this excitation source is the possibility of a bimodal population distribution; there is evidence for this in the $\mathrm{CF}_{2} \mathrm{Cl}_{2}$ and $\mathrm{CF}_{2} \mathrm{HCl}$ experiments at low levels of excitation $\left(<10000 \mathrm{~cm}^{-1}\right)$. The energy relaxation of the excited substrate at $\sim 300 \mathrm{~K}$ was monitored by: observing the propagation of the acoustic wave, observing the change in the gas density and detecting the infrared emission. The latter technique directly probes the substrate.

Experiments Probing the Acoustic Wave

The theory of time resolved optoacoustics (TROA) and thermal lensing was developed by Bailey et $\mathrm{al}^{2}$ and the experimental technique implemented by Gordon $\mathrm{et}^{\mathrm{al}^{3}}$. It is based on analyzing the initial acoustic wave 
which is produced when the internal energy of the substrate is transferned to the translational modes. The acoustic wave propagates radially from the excitation volume (a cylinder with a diameter on the order of $1 \mathrm{~mm}$ ) and is detected by a piezoelectric transducter located approximately $5 \mathrm{~cm}$ from the excitation volume. This acoustic wave is comprised of condensation (compression) and rarefraction (expansion) components; the amplitudes of these components are $I_{4}$ and $I_{\text {. }}$ respectively. Gordon ${ }^{4}$ has shown that this ratio is related to the V$T$ relaxation; by solving the continuity equations, assuming an excitation and relaxation function a calibration curve can be calculated. The shape of the acoustic wave is also an indicator of the relaxation function." We were unsuccessful in obtaining detailed information about the relaxation function by deconvoluting the observed wave form; calculations were consistent with an exponential decay.

The TROA data provide a $V-T$ relaxation time, $\tau$, for $\langle E\rangle$ such that $\mathrm{d}\langle\mathrm{E}\rangle / \mathrm{dt}=-1 / \tau\langle\mathrm{E}\rangle=-\mathrm{k}_{\mathrm{vT}}$ (E) $[\mathrm{NN}]\langle\mathrm{E}\rangle$

where $[N]$ is the concentration of colliders, $\langle E\rangle$ is the average energy and $k_{\mathrm{VT}}(E)$ is the phenomenological (energy dependent) bimolecular rate constant $(\mathrm{cc} /$ molec-sec). The change in $\langle\mathrm{E}\rangle$ is also related to the average energy removed per collision, $\langle\Delta E\rangle_{\Delta, 8}$ by

$\mathrm{d}<\mathrm{E}>/ \mathrm{dt}=-\omega<\Delta \mathrm{E}\rangle_{\mathrm{du}, \mathrm{B}}$ where $\omega$ is the collision frequency (for hard spheres $\omega=k_{\mathrm{HS}}[\mathrm{N}]$ and $k_{\mathrm{HS}}=\pi \mathrm{d}^{2}(8 \mathrm{kT} / \pi \mu)^{0.5}$ where $d$ is the collision diameter). Thus a collisional (collision per collision) efficiency, $\beta$, can be defined as $\beta(E)=k_{v T}(E) / k_{H s}=$ $\langle\Delta \mathrm{E}\rangle_{\mathrm{d}, \mathrm{B}}\langle\mathrm{E}\rangle$. The collisional efficiency is useful in that it factors out the collision cross section and the colliders reduced mass (which effects the relative velocity); thus $\beta$ is an intrinsic characteristic of the deactivator.

Approximately 650 experiments were performed with 11 substrates and 16 deactivators. Benchmark experiments with mixtures of $\mathbf{S F}_{6}$ and argon were in agreement with those reported by Gordon. The measured relaxation times were linear with pressure when the pressure changed by at least a factor of 4 ; this indicates that we are observing a bimolecular process. The average excitation energy was also varied from 1000 to 35000 $\mathrm{cm}^{-1}$. Except for the $\mathrm{CF}_{2} \mathrm{Cl}_{2}$ and $\mathrm{CF}_{2} \mathrm{HCl}$ systems with $\langle\mathrm{E}\rangle\left\langle\sim 10000\right.$, we found that $\mathrm{k}_{\mathrm{VT}}(\mathrm{E})$ and $\beta(\mathrm{E})$ were independent of $\langle E\rangle$; henceforth the energy dependence for these quantities will not be explicity indicated. The independence of $k_{V T}$ with energy indicates that $\langle\Delta E\rangle_{\Delta, \mathrm{B}}$ is linear with $\langle E\rangle$, i.e. the step size increase with the excitation energy. The hard sphere rate coefficients for the various collision partners and the experimentally determined collisional efficiencies are tabulated in Table I and Table II, respectively. To be noted is that $\beta \sim 3$ $10^{-3}$, or 3 collisions out of 1000 are effective in removing energy from the substrate; at $10000 \mathrm{~cm}^{-1}$ approximately $30 \mathrm{~cm}^{-1}$ of vibrational energy is removed per collision.

\section{Energy Dependence of $\mathrm{k}_{\mathrm{vT}}: \mathrm{CF}_{2} \mathrm{Cl}_{2}$ and $\mathrm{CF}_{2} \mathrm{HCl}$}

The average excitation energy per molecule was varied from 1000 to $35000 \mathrm{~cm}^{-1}$ using helium, neon, argon and krypton as deactivators; only translational energy can be transferred to these deactivators. The results of these experiments are displayed in Figure 2; the energy of one photon is approximately $1000 \mathrm{~cm}^{-1}$. Within experimental error these deactivators could not be distinguished and the deactivators are slightly more efficient in deactivating $\mathrm{CF}_{2} \mathrm{Cl}_{2}$ than $\mathrm{CF}_{2} \mathrm{HCl}$. Using a different technique, Setser ${ }^{6}$ has reported a rate coefficient that is $30 \%$ lower than what we observe; these results are consistent with known experimental errors. The effec: of decreasing $\beta$ when a halogen is replaced with an hydrogen atom was also observed in experiments we performed on the $\mathrm{CH}_{3} \mathrm{~F}$ system; $\beta$ for $\mathrm{CH}_{3} \mathrm{~F}$ is approximately an order of magnitude smaller than it is for $\mathrm{CF}_{2} \mathrm{Cl}_{2}$.

Both plots of Figure 2 indicate that below $10000 \mathrm{~cm}^{-1} \beta$ decreases with a decrease in $\langle E\rangle$. At $10000 \mathrm{~cm}^{-1}$ the density of internal energy eigenstates is approximately $10^{2}$ states $/ \mathrm{cm}^{-1}$ for $\mathrm{CF}_{2} \mathrm{HCl}$ and approximately $10^{4}$ for $\mathrm{CF}_{2} \mathrm{Cl}_{2} ;$ at $5000 \mathrm{~cm}^{-1}$ for $\mathrm{CF}_{2} \mathrm{HCl}$ intramolecular randomization of vibrational maybe slow. Intermolecular V-T relaxation for small polyatomics at low levels of excitation is thought to occur via the lowest vibrational modes; $\mathrm{Ln}\left[\mathrm{k}_{\mathrm{VT}}\right]$ is proportional to $\mathrm{V}_{\min }{ }^{7}$. From all considerations the vibrational frequencies for $\mathrm{CF}_{2} \mathrm{Cl}_{2}$ are lower than for $\mathrm{CF}_{2} \mathrm{HCl}$ : i. for $\mathrm{CF}_{2} \mathrm{Cl}_{2}$ the lowest frequency is $261 \mathrm{~cm}^{-1}$ as compared to $365 \mathrm{~cm}^{-1}$ for $\mathrm{CF}_{2} \mathrm{HCl}$, ii) the average vibrational frequencies for these molecules are 1130 and $642 \mathrm{~cm}^{-1}$, respectively, iii) $\mathrm{CF}_{2} \mathrm{Cl}_{2}$ has two vibrational frequencies below $400 \mathrm{~cm}^{-1}$ while $\mathrm{CF}_{2} \mathrm{HCl}$ only has one below $400 \mathrm{~cm}^{-1}$. The energy dependence of $\mathrm{k}_{\mathrm{vT}}$ may be due to a bimodal excitation function in which the groups have different characteristic rate coefficients so for this model $k_{\mathrm{VT}}($ observed $)=f(\langle E\rangle) k_{\mathrm{VT}}\left(\mathrm{E}_{1}\right)+(1-f(\langle E\rangle)) k_{\mathrm{VT}}\left(\mathrm{E}_{2}\right)$, where $\mathrm{f}(\langle\mathrm{E}\rangle)$ is the fraction of molecules with average energy $E_{1}$ when the overall average energy is $\langle E\rangle$. Although $f, E_{1}$ and $E_{2}$ were not calculated we observed that for a given fluence that the number of photons absorbed exhibited a pressure dependence; a good 
indication that a bimodal distribution was being generated.

Dependence of $\mathbf{k}_{\mathrm{vr}}$ on Substrate

The dependence of $\beta$ on substrate for argon as the deactivator is shown in Figure 3. For the series $C_{n} F_{2 n+2}$, $\beta$ monotonically decreases from 0.0075 to 0.0039 as $n$ increases from 3 to 8 . When $2 n+1 \mathrm{~F}$ atoms are replaced with $\mathrm{H}$ atoms for the $C_{n}$ molecule the efficiency increases; for $n=6, \beta$ increases from 0.0047 to 0.0095 when $F$ is replaced by $\mathrm{H}$. There is a comparable increase for the octane substrate. The large values of $k_{\mathrm{vT}}$ for these molecules prevented us from measuring rate coefficients for $n<6$ with this technique. The decrease of $\beta$ as $n$ increases was also observed for the monofluoro hydrocarbons.

The density of states for 1-fluoropropane is in excess of $10^{7}$ states $/ \mathrm{cm}^{-1}$ at $10000 \mathrm{~cm}^{-1}$; each additional $\mathrm{CH}_{2}$ group increases the density of states by a factor of $\sim 10^{3}$. Due to the lower vibrational frequencies the perfluorinated hydrocarbons will have an even higher density of states. Thus it is expected that these molecules will be in the quasicontinum at room temperature. Also to be noted is that for constant fluence irradiation these molecules did not exhibit a pressure dependence for the number of photons absorbed; it can be assumed that a bimodal distribution is not present. The lowest vibrational frequency for these molecules decrease from $\sim 150$ $\mathrm{cm}^{-1}$ to $<50 \mathrm{~cm}^{-1}$ in going from $\mathrm{C}_{4} \mathrm{H}_{10}$ to $\mathrm{C}_{8} \mathrm{H}_{18}$; the lowest frequency is reduced further as more fluorine atoms replace hydrogen.

The collisional efficiency for pentafluorobenzene $\left(\mathrm{C}_{6} \mathrm{~F}_{5} \mathrm{H}\right)$ a more rigid molecule than the linear chains (i.e. it does not have the low lying skeletal and torsional frequencies) was also determined. In spite of having 30 vibrational modes its efficiency was $\sim$ half that of $\mathrm{C}_{3} \mathrm{~F}_{8}$ with 27 modes or $\mathrm{C}_{4} \mathrm{~F}_{10}$ with 36 modes and comparable to $\mathrm{C}_{6} \mathrm{~F}_{14}$ with 54 modes. Thus it appears that the lower vibrational frequencies of the substrate facilitate energy transfer. This is also seen when comparing $\mathrm{C}_{3} \mathrm{~F}_{8}$ with the $\mathrm{CF}_{2} \mathrm{XCl}$ molecules; the lowest frequency in $\mathrm{C}_{3} \mathrm{~F}_{8}$ is less than the lowest in $\mathrm{CF}_{2} \mathrm{Cl}_{2}$ or $\mathrm{CF}_{2} \mathrm{HCl}$.

The observed decrease in $\beta$ with an increase in the number of vibrational modes can be understood by considering the energy dependence for the relaxation of a diatomic molecule. For a diatomic molecule the energy transfer rate coefficient increases linearly with an increase in excitation energy ${ }^{8}$. In the case of a homologous series of polyatomic molecules the vibrational frequency patterns are similar (see Figure 4). For a given total energy the average energy per oscillator decreases as the number of oscillators increases (this is true for both quantum or classical oscillators). The lowest vibrational frequency mode will have a decreasing average energy as the number of modes of the molecule increase; this produces a smaller $\mathbf{k}_{\mathrm{vr}}$. This effect and how the ratio of the average energy in a mode to the classical equiparition energy changes with the average energy is illustrated in Figure 5 and Figure 6, respectively. To be noted is that the average energy in low frequency modes vs total energy plots show negative curvature while the high frequency modes exhibit positive curvature; frequencies near the geometric mean show less curvature. An analogous argument can be used when $\mathrm{F}$ is replaced with $\mathrm{H}$; in this case the hydrogen substituted molecule has higher vibrational frequencies (the $1000 \mathrm{~cm}^{-1}$ C-F stretch is replaced with a $2970 \mathrm{~cm}^{-1} \mathrm{C}-\mathrm{H}$ stretch) which do not behave as efficient energy sinks. Thus, due to the quantum statistics (as shown in Figure 5 and Figure 6 classical equipartition of energy is not applicable at these level of excitation: see deviations from unity in Figure 6) the $\mathrm{C}_{n} \mathrm{FH}_{2 n+1}$ molecule has a smaller number of oscillators (energy sinks) than the $\mathrm{C}_{\mathrm{n}} \mathrm{F}_{2 n+2}$ molecule so that the average energy in the lowest frequency modes is increased. This increase produces an increase in $\mathbf{k}_{\mathrm{vr}}$.

Dependence of $k_{\mathrm{VT}}$ on Deactivator

A plot of $\beta$ as a function of the mass of the deactivator is shown in Figure 7 for the $C_{6} F_{14}$ and $C_{8} F_{18}$ substrates. Although this plot includes monatomic, diatomic and polyatomic deactivators there appears to be a general trend: the intrinsic collisional efficiency appears to have a broad maximum around 30-40 amu: a small decrease as the mass increases and a large decrease when the mass decreases to 2 amu. It is known that a decrease in reduced mass increases the relative velocity of the collision: this increases the collision rate and produces a more impulsive collision. The first effect has been taken care of in defining $\beta$. The second factor would suggest that $\beta$ increase as the reduced mass or the mass of the deactivator decreases. This increase is not observed; there must be another effect which explains the observed decrease. A billard ball model for energy transfer predicts that maximum efficiency for transfer exists when the masses of the colliding atoms are comparable; as these masses differ the efficiency decreases. Below $40 \mathrm{amu}$ the mass mismatch dominates and 
there is a decrease in $\beta$ with decreasing mas;; above 40 amu these two factors nearly balance one another and the ove:all effect on changing $\beta$ is small.

The effect of the deactivators structure on $\beta$ seems to be small; e.g. the efficiency of $D_{2}$ and He each with a mass of $4 \mathrm{amu}$ are comparable; this is also true of deactivators in the 28 to 32 amu range $\left(\mathrm{CO}, \mathrm{NO}, \mathrm{N} 2, \mathrm{C}_{2} \mathrm{H}_{6}\right.$ all have $\beta \sim 0.004$ while $\mathrm{O}_{2}$ has $\beta \sim 0.005$ ). It does not appear that $\mathrm{V}-\mathrm{V}$ transfer is important for this combination of substrate and deactivator. $\mathrm{CS}_{2}$ appears to show some anomaly; its efficiency is less than that of COS which is less than $\mathrm{CO}_{2}$. We do not have an explanation for this decrease.

\section{A Simple Model for $\mathbf{k}_{\mathrm{vr}}$ for Polyatomics}

The above observations indicate that there are two components to $\mathrm{k}_{\mathrm{vr}}$ : the lowest vibrational frequency (less than some critical value $\sim 200 \mathrm{~cm}^{-1}$ ) which acts as a doorway for V-T transfer and the average vibrational energy in that mode. Assuming that each mode relaxes independently a linear approximation results in $k_{v T}\left(E_{b a x}\right)=\Sigma \Sigma k_{v r}\left(m, E_{2}\right) f_{m}\left(E_{2}\right)$ where the summations are over all vibrational modes $m$ and valid energies for that mode which are less than the total energy $E_{200} f_{m}\left(E_{l}\right)$ is the fraction of molecules which have $E_{i}$ in mode $m$ and $k_{v_{T}}\left(m, E_{j}\right)$ is the rate coefficient for mode $m$ with energy $E_{i}$. If there is only one mode that provides the relaxation then the sums can be replaced by the product $k_{v T}\left(m, E_{z}\right) f_{m}\left(E_{2}\right), E_{z}$ is the statistical energy in the $m$ oscillator (lowest vibrational frequency). The first term can be calculated from $\mathrm{SSH}^{9}$ theory while the second term can be computed from the vibrational frequencies of the molecule ${ }^{10}$.

In the case of the $\mathrm{CF}_{2} \mathrm{XCl}$ molecules the dependence of $\mathrm{k}_{\mathrm{Vr}}\left(\mathrm{m}, \mathrm{E}_{2}\right)$ on $v$ determines the overall relaxation even though $\mathrm{f}_{\mathrm{m}}\left(\mathrm{E}_{2}\right)$ is larger for $\mathrm{CF}_{2} \mathrm{HCl}$ than it is for $\mathrm{CF}_{2} \mathrm{Cl}_{2}$. This is also the case for the substituted benzenes. For the homologous series of perfluorinated and monofluoro hydrocarbons, the dependence of the $\mathrm{k}_{\mathrm{VT}}\left(\mathrm{m}, \mathrm{E}_{2}\right)$ terms on $m$ are comparable and $k_{v T}$ is determined by $E_{1}$ (both the $f_{m}\left(E_{2}\right)$ and $k_{v T}\left(m, E_{2}\right)$ terms); however, both terms decrease as $\mathrm{E}$, decreases which is the case when the number of atoms increases or as the higher vibrational frequencies decrease. This model has the appropriate limits for small molecules at low and high levels of excitation and by assuming that there is rapid relaxation of internal energy for large polyatomic molecules accounts for our TROA observations.

\section{Experiments Probing the Gas Density: Heterodyne Experiments}

When the substrate's vibrational energy relaxes, the gas density of the energized volume changes. This change in gas density has been monitored by thermal lensing and interferometric techniques. Both techniques have the advantage of using fast photovoltaic detectors so that rapid relaxations can be monitored. Thermal lensing is a function of the change of the refractive index across the excitation area while interferometry relies only on a change of refractive index; both techniques integrate over the length of excited gas.

A Mach Zehnde: interferometer has been described in which extremely small changes in gas density can be observed." A CW He/Ne single frequency probe laser propagates through both arms of the interferometer; when the two beams combine an interference pattern results from the difference in optical path length of the two arms. A gas cell containing substrate and deactivator is placed in one arm of the interferometer such that a pulsed pump laser propagates in line with the He/Ne laser. The change in gas density indirectly produced by the pump laser causes a change in the optical path so that the intensity of the interference pattern changes. The intensity at the detector is proportional to the sin of the phase change; a son-linear and non-monotonic function.

This non-monotonicity can cause problems in interpreting the direction for changes in the optical path, i.e. gas density in our experiments. Specifically, if the change in optical path exceeds an integral number of $\lambda_{\text {itendo }} / 2$ then the change becomes ambiguous. The non-linearity of the signal with path difference requires a reproducible starting point if signal averaging is to be used as is the case for these small signals. Additionally, the starting point for each pulse should occur at a path difference which will generate the largest signal. A given change in path length produces a large change in signal when the phase difference is 0 or $\pi$ than if it $w s i \pi / 2$

(constructive interference) or $3 \pi / 2$ (destructive interference). For example, a change of phase shift of $1^{\circ}$ gives a signal change 115 times larger if the starting phase is $0^{\circ}$ than if it were at $\pi / 2$.

We have modified and stabilized the traditional Mach Zehnder interferometer so that absolute phase shifts can be measured in a signal averaging mode with enhanced sensitivity; a schematic layout is shown in Figure 3. Retroreflections into the $\mathrm{He} / \mathrm{Ne}$ cavity were eliminated by piacing a Bragg cell between the $\mathrm{He} / \mathrm{Ne}$ polarized laser 
and the entrance to the Mach Zehnder interferometer; the Bragg cell upshifts the frequency of the He/ $\mathrm{Ne}$ by 80 MHz. A quarter wave plate was placed in one arm of the interferometer and two photovoltaic detectors with polarizers oriented $90^{\circ}$ to each other were placed at equivalent outputs of the interferometer. In effect the interferometer is two interferometers; one measuring the interference of two beams with an unknown phase difference $(\Delta \phi)$ an the other with a phase shift of $\Delta \phi+\pi / 2$. Thus the signals are in quadrature and absolute direction can be derived: $\tan (\Delta \phi)$ is the ratio of the two signals. The signal from one of the detectors is directed to a lockin amplifier which provides a correction and modulation signal $(508 \mathrm{~Hz})$ to a piezoelectric device attached to one of the mirrors in the interferometer. One arm of the interferometer is controlled so that the resulting signal is at a miminum; one detector locks in to an optical path difference corresponding to destructive interference while the other detector is offset by $90^{\circ}$, i.e. at $0^{\circ}$ or $270^{\circ}$. To prevent contamination of the phase shift from the relaxation by the servo loop; the stabilizer is turned off during the relaxation; this amounts to approximately 30 msecs every 2 seconds (which is the cycle time for the pump laser).

The reproducibility of the starting point is verified by the fact that the signal to noise ratio scales with the number of pulses which are averaged; if the starting point was random a degradation in signal to noise would be observed with the number of pulses. The measurement of absolute phase was also tested; a repetitive voltage ramp was supplied to the piezoelectric device so that there was an optical path increase of 20 microns followed by a decrease of 20 microns. The two detector signals were processed and produced a plot of the change in optical path vs of time which correlated well with the voltage ramp and specifications for the piezoelectric device.

Relating the Difference in Optical Path to Changes in the Gas Density

In a similar manner as to what we did for the TROA experiments the conditions were set so that the time for the acoustic wave to traverse the diameter of the pump laser was less than the V-T relaxation time; this time was less than the charactertics times for thermal conductivity and mass diffusion. The continuity equations can solved for the change in gas density in the excitation volume ${ }^{2}$. A family of curves result when the density. change is plotted as a function of time for various $k_{v r}$ 's (see Figure 9).

The observed phase shift, $\Delta \phi$, is proportional to a change in temperature, $\Delta T$, and the cell length. If the relaxation is exponential then $\Delta \mathrm{T}$ will follow the equation

$\Delta T(t)=\Delta T_{\max }\left(1-e^{-t w n t}\right)$

so that the observed signal at each detector is

$S_{i}(t)=A_{i} \sin \left(c \Delta T(t)+\phi_{i 0}\right)+B_{1}$

[ $\phi_{i 0}$ is the phase shift before initiating the relaxation and $\phi_{10}-\phi_{20}=\pi / 2$ ]

To be noted is the high degree of nonlinearity of these functions and that they are not monotonic. A nonlinear least squares program was written to find the optimum value of $\tau$. A mixture of $\mathrm{C}_{2} \mathrm{H}_{4}$ diluted in argon was used for benchmark experiments. The signals observed for one of these experiments and the best fit curve are shown in Figure 10. A value of $410^{-13} \mathrm{cc} / \mathrm{molec}^{-\mathrm{sec}}$ for $\mathrm{k}_{\mathrm{VT}}$ was determined; this is in fair agreement with $210^{-13}$ $\mathrm{cc} /$ molec-sec reported by Flynn ${ }^{12}$ using the thermal lensing technique. This difference may be due to difference in the average energy of excited $\mathrm{C}_{2} \mathrm{H}_{4}$.

Although only six experiment have been completed, the results are encouraging. The technique has the advantage of using fast photodiodes so that fast relaxations can be observed. We are hopeful that the shape of the relaxation will provide more information on the energy dependence of $\langle\Delta \mathrm{E}\rangle_{\mathrm{ul}}$.

\section{Experiments Probing the Infrared Emission}

Barker ${ }^{13}$ has extended infrared fluorescent techniçues to monitor energy relaxation of azulene and benzene type molecules. For these experiments the fundamental emission of a particular vibrational mode (i) is monitored; the band of $n \rightarrow n-1$ transitions are not resolved as a function of $n$. Thus the emission rate of that mode is a sum of emissions from various excited levels; Durana and McDonald ${ }^{14}$ have derived an expression for the emission intensity as a function of total energy. By assuming that the Einstein coefficient for emission from state $n$ is proportional to $n$ (this was aiso assumed by McDonald) it can be shown that the emission intensity is also proportional to the average vibrational energy of that mode $\left(\left\langle\mathrm{E}_{i}\right\rangle_{j}\right)$. Assuming that the total energy of the molecule is statistically distributed amongst its vibrational modes then $\left\langle E_{1}\right\rangle_{\text {, }}$ is a measure of $\langle E\rangle_{\text {. }}$. Due to the quantum nature of the vibrational frequencies and their distribution the ratio $\left\langle\mathrm{E}_{\rangle}\right\rangle \mid\langle\mathrm{E}\rangle$ is a function of 
- E>, i.e. classical equipartition of energy between modes is not valid (see earlier discussion and Figure 5 and Figure 6). Using all the vibrational frequencies for the emitter, a calibration curve, $\mathrm{I}(\langle\mathrm{E}\rangle)$, can be calculated so that the monitored intensity, $I_{4}$ can be transformed to $\langle E\rangle_{\text {i }}$. For a given pressure the number of collisions that the substrate undergoes is known so that in theory $\langle\Delta \mathrm{E}\rangle_{\mathrm{B}}$ for that specific population can be computed.

The apparatus used for these experiments is shown in Figure 11; the C-H stretching frequency $\left(2970 \mathrm{~cm}^{-1}\right)$ was monitored. Potential problems for these experiments are the low emission rates (due to the small Einstein probabilities and the low fraction of available energy which is distributed in the C-H stretch), the low sensitivity, high thermal background and micmsecond time constants for detectors in this spectral region. These deficiencies are reduced by extensive signal averaging.

Results from these experiments for the chain like molecules with $\mathrm{C}-\mathrm{H}$ stretches $\left[\mathrm{CH}_{3} \mathrm{~F}, \mathrm{CF}_{2} \mathrm{HCl}\right.$ and $\mathrm{C}_{\mathrm{n}} \mathrm{H}_{2 n+1} \mathrm{~F}$ $(n=2-8)$ ] were difficult to obtain and interpret as contrasted to Barkers ${ }^{13}$ experiments with pancake geometry. The intensity-time profiles exhibited an overall biexponentia! decay with small undulations; these undulations were pressure dependent. We have interpreted this behavior as due to: to gas density fluctuations initiated by the energy relaxation, a heterogeneous spatial distribution of excited substrate which exhibits a time dependence, and the dependence of emission detection efficiency on the emitters position. Additional complications are due to the diffusion of substate from the observation and excitation volume. Although we will continue to analyze these experiments to understand what is happening; it appears that we will not obtain useful energy transfer information.

\section{Computer Experiments: Simulating Energy Relaxation}

An insight on energy relaxation can be obtained by trajectory calculations in which the microscopic details of the encounter are observed. Computer experiments were designed in which the rotation, vibration and relative translational energies were specified and the outcome was recorded. The trajectories were produced by integrating the classical equations of motion; the VENUS computer program developed by Hase and coworkers was used ${ }^{15}$.

Specifically the process

$$
A\left(E_{r a d} E_{v i b}\right)+M\left(E_{t r m}\right) \rightarrow A\left(E_{r o s}^{\prime} E_{v i b}^{\prime}\right)+M\left(E_{t r n}^{\prime}\right)
$$

was simulated where $A$ is $C X_{4}$ and is $M$ a structureless particle. In these calculations the mass of $M$ and $X$ was systematically varied; the initial vibration, rotation and relative translational energies were held constant. The atomic interaction potential (intramolecular and intermolecular) was kept constant such that a change in mass directly influenced the vibrational frequency and the moments of inertia of the substrate. The masses also affect the relative velocity; the relative velocity decreases with an increase in the reduced mass of the collision partners. The specific system parameters which were used are presented in Table III. To be noted is that the moments of inertia increase by a factor 20 in going from $\mathrm{CH}_{4}$ to $\mathrm{CF}_{4}$ while the effective vibrational frequency decreases by a factor of three. The density of vibrational energy eigenstates at $100 \mathrm{kcal} / \mathrm{mole}$ (the initial vibrational energy) increases by nearly $10^{4}$ when going from $\mathrm{CH}_{4}$ to $\mathrm{CF}_{4}$.

The rate of energy transfer was calculated from the expression ${ }^{16}$

$$
\operatorname{Rate}_{\mathrm{B}, \mathrm{a}}=\operatorname{Limit}(\mathrm{d} \rightarrow \infty)\left[(8 \mathrm{kT} / \pi \mu)^{0.5} \pi \mathrm{d}^{2} \Sigma\left(\Delta \mathrm{E}_{i}^{\mathrm{p}} / \mathrm{N}\right)\right]=\mathrm{Z}_{\mathrm{c}}\left\langle\Delta \mathrm{E}^{\mathrm{m}}\right\rangle_{\mu}
$$

where the sum is over the $\mathrm{N}$ calculated trajectories and $\Delta \mathrm{E}_{\mathrm{i}}^{\mathrm{n}}$ is the energy transferred for the specified trajectory raised to the $n^{\text {th }}$ power. The energy transfer rate and $Z_{0}$ (crmputed from an assumed cross section: $\pi \mathrm{d}^{2}$ ) are used to calculate $\left\langle\Delta \mathrm{E}^{\mathrm{a}}\right\rangle_{\mathrm{dl}}$ for each energy sink/source: translations, rotations and vibrations. The results of these calculations are presented in Table IV, Figure 12, Figure 13 and Figure 14. The interpretation of these results is from a classical mechanics framework; application to real molecules may not be valid as discussed in a later paragraph relating to zero point energy.

Although there are some minor anomalies, the amount of vibrational energy transferred from $\mathrm{CX}_{4}$ increases as the mass of deactivator decreases from 40 to $0.5 \mathrm{amu}$. In this case the collision becomes more impulsive as the mass decreases; the collision time is reduced by the increased relative velocity of the smaller reduced mass of the collision partners. Various collisional energy transfer models predict that the amount of energy transferred will increase as the collision becomes more impulsive. This is the reason for the overall increase in $\langle\triangle \mathrm{E}\rangle_{\text {vib }}$ for $\mathrm{CH}_{4}$ and $\mathrm{CD}_{4}$ with decrease in the mass of deactivator. It is seen that the energy transferred is vibration to translation, i.e. as the vibrational energy tansferred increases so does the resulting change in translational energy. 
For comparable collision times, classical collision mechanics predicts that the efficiency for transfer will decreases as the difference in masses of the colliding atoms increases. The effect of mass defect can be seen for the $\mathrm{CF}_{4}$ system; when the mass of the deactivator is below $4 \mathrm{amu}$, the amount transferred decreases as the deactivator mass decreases. Thus we are seeing at 4 amu a competition between efficient impulsive collisions and the ineffiency of a light collider with the mass $19 \mathrm{~F}$ atom in $\mathrm{CF}_{4}$. This competition would not be effective for the $\mathrm{CH}_{4}$ and $\mathrm{CD}_{4}$ systems until the mass of deactivator is less than $0.5 \mathrm{amu}$; our calculations had a lower limit of $0.5 \mathrm{amu}$ for the deactivator. However, it is expected that the competition will appear at a higher mass for $\mathrm{CD}_{4}$ than for $\mathrm{CH}_{4}$.

Deactivators with masses $>4$ amu remove larger amounts of vibrational energy as the mass of the substrate increases, i.e. more energy is removed from $\mathrm{CF}_{4}$ than $\mathrm{CH}_{4}$. In going from $\mathrm{CH}_{4}$ to $\mathrm{CF}_{4}$ the vibrational frequency decreases so that for a given relative velocity the heavier molecule will have a more impulsive collision and more vibrational energy will be transferred. Due to the increase in the difference of masses between collision partners (mass mismatch) this trend is reversed for deactivator masses $<4$ amu. However, for masses less than 4 amu the analysis for $\mathrm{CH}_{4}$ and $\mathrm{CD}_{4}$ is even more complex: rotational coupling (vibration to vibration/rotational energy transfer) becomes important. For $\mathrm{CH}_{4}$ the rotational energy transferred decreases with a decrease in deactivator's mass; for $\mathrm{CD}_{4}$ the reverse effect is observed while for $\mathrm{CF}_{4}$ there is no correlation.

The above analysis considered the first moment of the transition probabilities, i.e. $\langle\Delta E\rangle_{\mu l} ;\langle\Delta E\rangle_{\mu l}$ is usually sufficient to parameterize weak collider rate coefficier:ts. However, the second moment, $\left\langle\Delta \mathrm{E}^{2}\right\rangle_{\mu \nu}$, also provides some insight as to the complexity of the form of the energy transfer function and may be required for understanding experiments which do not monitor highly averaged quantities. The second moment relates to the breadth of the distribution function so that $\left\langle\Delta \mathrm{E}^{2}\right\rangle^{0.5}{ }_{\text {al }} /\left\langle\Delta \mathrm{E}_{\mathrm{all}}(=\gamma)\right.$ can be used as an indicator as to the shape of the transition probabilities. It can be shown for a single transition (either up or down) $\gamma=1$, for an exponential distribution $\gamma=1.41$, for mustiexponential models (both up and down transitions) $\gamma>1.41$. It is seen that $\gamma_{\text {wib }}$ is between -1.6 and -2.8 for all three systems with a slight dependence on deactivator mass; the $\mathrm{CH}_{4}$ system is broader than the $\mathrm{CD}_{4}$ which is broader than the $\mathrm{CF}_{4}$ system. The rotational energy distributions are wider distributions than those observed for vibration; $\gamma_{\text {ra }}$ goes from a nearly constant of 3 for $\mathrm{CF}_{4}$ (mass 9 being 5 ) to $\mathrm{CD}_{4}$ ranging from -40 to +10 while for $\mathrm{CH}_{4}$ the range is between +3 and +10 . These wild variations in $\gamma$ may be an indicator that rotational resonances exist in which the molecular rotation and orbital angular mometum efficiently interact.

As noted earlier a problem associated with factoring the rate of energy transport into a collision rate and $\langle\Delta \mathrm{E}\rangle_{\nu \mu}$ is in assigning the correct collision cross section. Traditionally the cross section has been set equal to the Lennard-Jones $\sigma$. This is not important in our internal comparisions but is crucial in comparing external systemis (experimental or simulated). Our trajectory calculations show that the true cross section for energy transfer is substantially larger than $\sigma_{\mathrm{LJ}}$. In Figure 14 the scattering angle as a function of impact parameter is shown; when the impact parameter is approximately equal to $\sigma_{\mathrm{L}}$ the scattering angle is nearly zero. However, with increasing impact parameter the scattering angle increases, reaches a maximum and then decreases to zero as the impact parameter increases; as expected, for very large impact parameters the scattering angle is zero. Clearly the collision cross section is greater than $\sigma_{\mathrm{LJ}}$. This can also be seen when $\langle\Delta E\rangle_{\text {all }}$ is plotted vs the impact parameter; $\langle\Delta E\rangle_{\mu \mu}$ converges at an impact parameter substantially greater than $\sigma_{L j}$. In all cases the calculations indicated converged values for the $\langle\Delta \mathrm{E}\rangle_{\text {all }}$ quantities.

Known problems with classical mechanical calculations are the quantized energy levels for the oscillators and a non-zero zero point energy for each oscillator ${ }^{17,18}$. A partial solution to the first problem is to partition the final vibrational energy from each trajectory into a vibrational energy state in which upper and lower energy limits are defined for each state. This pseudo-quantization of energy levels becomes a better approximation as the number of modes for a molecule increases. The classical trajectories also maintain an equiparition of energy in the normal modes; as shown in Figure 5 and Figure 6 this is not the case for frequencies that are not small relative to the total energy. The second problem relating to the handling of zero point energy is also complex; however, a solution has been presented but not tested for large molecules. For the classical calculation, the total vibrational energy is allowed to freely migrate between the various modes; through out the trajectory, even though the total zero point is constrained, some oscillators may have energies less than their zero point energy. However, quantum mechanically the zero point of each oscillator must be constrained to be equal to or greater than its zero point energy. 
The consequences for inclusion for this zero point energy effect increases with molecular complexity; for hydrocarbons there is an increase in zero point energy of approximately $20 \mathrm{kcal} / \mathrm{mole}$ per $\mathrm{CH}_{2}$ group. For small molecules the effect of zero point may be small; the zero point energy for $\mathrm{CH}_{4}$ is $\sim 25 \mathrm{kcal} / \mathrm{mole}$ and decreases to $\sim 15 \mathrm{kcal} /$ mole for $\mathrm{CF}_{4}$. Although this difference is less than the addition of a $\mathrm{CH}_{2}$ group it may be important. Due to the unknown effect of properly including zero point energy (i.e. freezing the zero point energy of each oscillator) we did not extend our calculations to the $C_{2} X_{2}, C_{2} X_{4}, C_{2} X_{6}, C_{3} X_{8}$ and $C_{4} X_{10}$ analogs. The importance of not freezing the zero point energy on the calculated $\langle\Delta E\rangle_{\mu}$ quantities for the $C X_{4}$ systems is not known. $A$ third problem arises when the energy dependence of $\langle\Delta E\rangle_{\Delta}$ is calculated. When the total classical vibrational energy is $E_{v i b}$, what is the effective vibrational energy: $E_{v i b}$ or $E_{v i b}$ - total zero point energy? More work must be done to describe the corrections that must be made to the classical calculations. As a consequence of these problems, only gross trends in $\langle\Delta E\rangle_{\Perp}$ should be considered when application of classical calculations are made to real systems. 


\section{HIGH TEMPERATURE COMPLICATIONS fOr UNIMOLECULAR REACTIONS}

At low temperature the quasi-steady state approximation is made for unimolecular reactions and various energy transfer corrections are used to modify strong collider calculations. However, for certain high temperature unimolecular systems a steady state or quasi steady state is not obtained and a time-indep ident rate coefficient cannot be defined. Unfortunately, there have been occasions in which the low temperatire parameters have been applied wh high temperature systems; due to the unknown limitiations of the low temperature formulations incorrect conclusions were reported. Two specific cases are the steady state approximation and the weak collider modification. It is important that practioners and modelers that use these approximate equations are aware of their limitations. Unfortunately, the limitations of these equations are often not explicitly delineated; this is due mainly to the fact they are often not comprehensively tested over a wide range of experimental conditions. The following work was undertaken to provide a reference for some of the deviations which occur at high temperatures. This section is divided into two parts: the strong collision ${ }^{19}$ corrections and L.e weak collider ${ }^{20}$ modifications that must be made to the strong collision results. These considerations are particularly relevant in high temperature systems such as shock tubes, flame and combustion.

\section{High Temperature Devintions from the Approximate RRKM Expression}

For the first order unimolecular reaction,

$$
\text { A } \rightarrow \text { Products }
$$

the rate coefficient, $k_{\mathrm{ww}}$ is given by $-1 /[\mathrm{A}] \mathrm{d}[\mathrm{A}] / \mathrm{dt}$. The Lindemann scheme provides a simplified three step mechanism which involves: activation, deactivation and reaction, as depicted below.

$$
\begin{array}{lc}
A+M \rightarrow A^{*}+M & k_{1}: \text { activation } \\
A^{*}+M i A+M & k_{1}: \text { deactivation } \\
A^{*} \rightarrow \text { Products } & k_{2}: \text { reaction }
\end{array}
$$

Assuming a steady state for $A^{*}$ gives $k_{\text {ani }}=k_{1} k_{2}[M] /\left(k_{1}[M]+k_{2}\right)$. At high pressure $k_{\text {wi }}$ reduces to $k_{2}$ while in the low pressure limit $t_{-i}=k_{1}[M]$, i.e. the rate coefficient is the collision rate to produce $A^{*}$. This model accounts for the limiting regions and the general falloff but is deficient in quantifying the shape and pressure region of the falloff. For $k_{\text {mai }}$ to be calculated properly, more energy levels must be included and account must be taken of the probabilities of transporting a molecule from any total energy state i to any total energy state 1 . Since for large polyatomic molecules the density of states is very high, the molecular states are grained together into pseudo energy levels and a detailed master equation is written whereby the population of each energy level is replenished and depleted explicity

$$
\begin{gathered}
\frac{d(A(E, t)]}{d}=-\int_{0}^{-} Z(E)[M] P\left(E^{\prime}, E\right)[A(E, t)] d E^{\prime}+\int_{0}^{-} Z\left(E^{\prime}\right)[M] P\left(E, E^{\prime}\right)\left[A\left(E^{\prime}, t\right)\right] d E^{\prime}-k(E)[A(E, t)] \\
\frac{d(A(E, t)]}{d}=-\int_{0}^{-} R\left(E^{\prime}, E\right)[M][A(E, t)] d E^{\prime}+\int_{0}^{-} R\left(E, E^{\prime}\right)[M]\left[A\left(E^{\prime}, t\right)\right] d E^{\prime}-k(E)[A(E, t)]
\end{gathered}
$$

where $R\left(E^{\prime} E\right)=P\left(E^{\prime}, E\right) Z(E)$ is the rate coefficient for energy transfer and $Z(E)[M]$ is the number of collisions per unit time. The exact form of $P\left(E^{\prime}, E\right)$ or $R\left(E^{\prime}, E\right)$ is not known and various benchmark models have been invoked: stepladder, gaussian, poisson and exponential. These coupled differential equations can be solved once the $R\left(E^{\prime}, E\right)$ and $k(E)$ are known; eigenvalue equation techniques can be used using the probability and rate constant matrices. In general, $[A(E, t)]=\Sigma a_{i}(E) e^{-k(j) t}$ where $A_{i}(E)$ are the energy dependent normalization factors and $k(j)$ are the negative eigenvalues. If one of the $k(j) ' s(=k)$ is much smaller than the rest then $[A(E, t)]$ is given by $a(E) e^{-k}$ so that $k_{\text {, }}$ becomes $k$.

For a quasi steady state $d([A(E, t)] /[A(t)]) / d t=0$ so that $d[A(E, t)] / d t=-k_{\text {ani }}[A(E, t)]$ and equation $(1)$ gives

$$
-\int_{0}^{\infty} Z(E)\left[M P\left(E^{\prime}, E\right)[A(E, t)] d E^{\prime}+\int_{0}^{\infty} Z\left(E^{\prime}\right][M] P\left(E, E^{\prime}\right)\left[A\left(E^{\prime}, t\right)\right] d E^{\prime}-K(E)[A(E, t)]=-k_{\text {mand }}[A(E, t)]\right.
$$

For the case of a strong collider $P\left(E, E^{\prime}\right)=B(E)$ and we obtain 


$$
k_{\text {id }}^{\infty}=\int_{0}^{-} \frac{Z[M] B(E) k(E)}{Z[M]+k(E)-k_{\text {mid }}^{\infty}} d E
$$

Most expressions that have been used for $k_{\text {ani }}{ }^{\infty}$ have ignored the $-k_{a x i}{ }^{\infty}$ term in the denoniinator, appropriately this will be desigrated as $k_{\text {men }}^{\infty}(a p p)$.

$$
k_{\min }^{c}(\operatorname{app})=\int_{0}^{\infty} \frac{Z[M] B(E) k(E)}{Z[M]+k(E)} d E
$$

In the high pressure limit, $k_{\infty}{ }^{\infty}$ is just $\int B(E) k(E) d E$; in this case the $-k_{\text {ani }}{ }^{\infty}$ term in the denominator is negligible and does not contribute. Both expressions illustrate that collisions do not play an active role in the reaction at high pressure so that the sc superscript can be removed. In the low pressure limit $k_{0}{ }^{\infty}$ is $\int Z[M] B(E) k(E) d E /(k(E)$ $\left.\mathbf{k}_{0}{ }^{2}\right)$. At this limiting pressure, collisions do play a role and a wc superscript is needed when applicable; the subscript 0 indicates the limiting low pressure value.

For low temperatures, $k(E)>>k_{0}{ }^{\circ}$ and the exact equation (3) yields the conventional expression $k_{0}{ }^{c}=\int Z[M] B(E) d E$

and $k_{0}{ }^{\alpha}$ approximates Arrhenius behavior.

At high temperatures the last term in the ciencminator of equation (3) is not negligible, and even a quasi steady state is not obtained. Montroll and Shuler ${ }^{21}$ realized that a quasi steady state is not obtained and introduced a lower bound for E $/$ RT below which steady state is not applicable. Although solutions for solving the exact equation have been pr:blished ${ }^{22}$ an operational lower bound for acceptable solutions has not been reported. We set out to determine the parameters which are important in specifying this lower limit so that some simple diagnostics could be used to determine when equation (4) could be used in place of the exact equation (3).

Calculations were performed ior two molecules: cyclobutane and quadricyclane under a variety of pressures and temperatures. $k_{2 x i}{ }^{\times}$was computed using both equations (3) and (4). The results are expressed as percent relative deviations from the approximation, i.e $\Delta=100\left[k_{u n i}{ }^{\circ}(a p p)-k_{u n i}{ }^{\infty}\right] / k_{a n i}{ }^{* c}$ (app). Of the two molecules chosen, quadricyclane has 39 normal modes and an activation energy of $33.5 \mathrm{kcai} /$ mole and represents an isomerization re..un. Cyclobutane has a smaller number of normal modes (24), a higher activation energy $(64.7 \mathrm{kcal} / \mathrm{mole})$ and represents : fission reaction. In addition, for each molecule the activation energy was lowered or raised artificially to evaluate separately the effects of the number of normal modes and threshold energy. The falloff for each molecule is also a variable and can be parameterized by the reaction order, $\Phi$. The reaction order is defined as

$\Phi=d \ln \left[k_{\text {ani }}{ }^{\alpha}\right] / d \ln [\omega]+1$

where $\omega=Z[M]$ is the collisional frequency. Each molecule is discussed separately and some general conclusions are made.

Figure 15 shows $\Delta$ as a function of pressure for temperatures between 1500 and $2500 \mathrm{~K}$ for quadricyclane. To be noted is that the rate coefficient for the exact expression is always larger than that calculated from the the approximate equation. The deviation decreases with decreasing temperature and the maximum deviation occurs at lower pressure as the temperature is decreased. Figure 16 illustrates the deviation as a function of $\Phi$; in this plot the maximum deviation occurs between reaction order of 1.7 and 1.9, (very close to the second order region). For reaction order of 1.7 at $2500 \mathrm{~K}$ the deviation is $-40 \%$ and it is only $\sim 15 \%$ at $1500 \mathrm{~K}$. At other reaction orders the deviation is not negligible. For reaction order of 1.5 at $2500 \mathrm{~K}$ the deviation is $\sim 35 \%$ and the deviation approaches 0 slowly as the reaction order goes to 1.0. This is important since it shows that the approximate equation is not acceptable at high temperatures even at $\Phi$ close to 1.0 . The falloff curve is not only shifted but there is a change in its shape. The lower the temperature, the smaller the deviation and the span of the falloff is decreased.

To study the effect of threshold energy $E_{o}$ on the deviation, the value of $E_{0}$ was raised arbitrarily to 64.7 
$\mathrm{kcal} / \mathrm{mol}$ which is the value of $\mathrm{E}_{0}$ of cyclobutane. The results indicate that the maximum of the deviation is shifted to lower pressures and the absolute value of the deviation is reduced. For $2500 \mathrm{~K}$ the change is from $-40 \%$ to $-18 \%$, more than a factor of 2 . The region of deviation is shifted to higher reaction orders; between 1.82 and 1.92. The dependence of order as a function of pressure is broader and falloff appears at lower pressure. The net effect of higher threshold energy is to red'sce the deviation, shift the maxinum of the deviation toward the second order region and shift the second order region to lower pressures. Nevertheless the deviation at high pressure ( $>10^{4}$ torr) is still significant.

Cyclobutane has a lower number of normal modes (24) than quadricyclane (39) and higher threshold energy (64.7 vs $33.5 \mathrm{kcal} / \mathrm{mole})$. The deviation is smaller than what was observed for quadricyclane; the maximum deviation is $\sim 10 \%$ at $2500 \mathrm{~K}$ and almost nonexistent at $1500 \mathrm{~K}$. The higher the temperature, the higher the pressure of the maximum deviation. The maximum deviation occurs in the range of $1.8<\Phi<1.9$, i.e. near the second orde: region. The deviation plot is broad and it declines to zero (for $2500 \mathrm{~K}$ ) only in the first order region. Reducing $\mathrm{E}_{\mathrm{o}}$ arbitrarily to $33.5 \mathrm{kcal} / \mathrm{mole}$ (the threshold value of quadricyclane) causes major changes. The maximum deviation is shifted to higher pressures and it is increased from $\sim 10 \%$ to $\sim 28 \%$. The maximum deviation occurs at lower values of the reaction order compared to the previous high $\mathrm{E}_{\mathrm{o}}$ case. A shift in $\Phi$ to higher pressures is produced such that the high pressure region will not be attainable in practice.

\section{Conclusion}

It is clear from the information presented above that the conventional but approximate expression for $\mathrm{k}_{\mathrm{enn}}{ }^{\mathrm{c}}$ is applicable at lower temperature but can lead to large errors at high temperatures. This is also true when $k_{\text {wox }}{ }^{\infty}$ is calculaied by using an effective collision number; this is sometimes done for weak colliders by introducing a collision efficiency. Specific conclusions are:

i. large molecules always have larger deviations from the approximate equation than smaller molecules

ii. deviations increase with a decrease in critical threshold

iii. for smaller values of $E_{0}$ the maximum deviation shifts to higher values of the pressure compared to the case for a higher value of $E_{0}$

iv. as the temperature decreases the size of the deviation decreases.

The above four conclusions and our calculations lead to two practical rules of thumb:

a) the error in using the approximate equation is negligible for EdRT $>20$ and b) yery roughly and based on limited data for $E_{\delta} / \mathrm{RT}<17$ the deviation is proportional to the number of vibrational modes of the reactant. These conclusions should be helpful to practioners and modelers of combustion chemistry that use published equations in which limitations are not explicitly given.

\section{Weak Collision Considerations}

The master equation (1) defined above is general and can be solved for any collisional model for weak collisions; however, the equations cannot be simplified and decoupled as was the case for a strong collider. In general, analytical solutions are not available and the populations must be determined by numerical methods. For weak colliders the populations for energy levels less than $E_{o}$ are depleted. This depletion and the short energy reach of a weak collider produce observed rate coefficient which are smaller than that for a strong collider. The depletion increases as the reaction order increases. To avoid performing master equation calculations for each chemical system various corrections have been suggested and used. A collisional efficiency

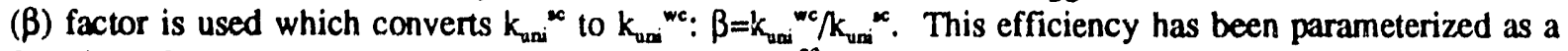
function of temperature and average step size by Troe $^{23}$

$$
\frac{\beta_{0}}{1-\beta_{0}^{0.5}}=\frac{-\langle\Delta E\rangle_{\text {ell }}}{F_{E} R T}
$$

and by Tardy and Rabinovitch ${ }^{24}$

where the $\langle\Delta E\rangle_{\alpha}$ and $\langle\Delta E\rangle_{d}$ quantities are the average energy removed per collision and the average energy 


$$
\beta_{0}=\frac{\left\langle\Delta E s_{d l^{2}}^{2}\right.}{(R T)^{2} I_{R}}
$$

removed per down collision; these averages can be calculated from the probability model by

$$
\begin{aligned}
&<\Delta E\rangle_{d}= \frac{\int_{0}^{E^{\prime}}\left(E-E^{\prime}\right) P\left(E, E^{\prime}\right)}{\int_{0}^{\Delta^{\prime}} P\left(E, E^{\prime}\right) d E} \\
&\langle\Delta E\rangle_{\alpha]}=\frac{\int_{0}^{\infty}\left(E-E^{\prime}\right) P\left(E_{,} E^{\prime}\right) d E}{\int_{0}^{-} P\left(E, E^{\prime}\right) d E}
\end{aligned}
$$

In the case of a simple step ladder model (only a single energy jump is allowed) $-\langle\Delta \mathrm{E}\rangle_{\mathrm{d}}=\langle\Delta \mathrm{E}\rangle_{\mathrm{d}}{ }^{2} /\left(\langle\Delta \mathrm{E}\rangle_{\mathrm{d}}+\mathrm{RT}\right)$.

However, for models that involve more than a single step the variation of the density of states of the molecule becomes important. Troe has added a correction term $\left(\mathrm{F}_{\mathrm{B}}\right)$ so that

$$
\begin{aligned}
-\langle\Delta E\rangle_{d u} & =\frac{\langle\Delta E\rangle_{d}^{2}}{\langle\Delta E\rangle_{d}+F_{z} R T} \\
\text { where } \quad F_{E} & =\frac{\int_{0}^{-(E) / \rho\left(E_{0}\right) e^{-\left(E-E_{0}\right) / E r_{d E}}}}{R T}
\end{aligned}
$$

while Tardy and Rabinovitch (TR $)^{25,26}$ have introducted an effective temperture $\left(T_{e}\right)$ which is depends on the ambient temperature $(T)$ and the inversion temperture $\left(T_{l}\right.$, which is a function of molecular properties).

$$
\begin{gathered}
-\langle\Delta E\rangle_{\text {all }}=\frac{\langle\Delta E\rangle_{d}^{2}}{\langle\Delta E\rangle_{d}+R T_{e}} \\
\text { where } T_{a}=\frac{T}{1-\frac{T}{T_{l}}}
\end{gathered}
$$

and 


$$
\begin{aligned}
T_{l} & =\frac{\langle\Delta E\rangle_{d}}{R \ln \left[\rho\left(E+\langle\Delta E\rangle_{d}\right) / \rho(E)\right]} \\
& =\frac{E}{R\left[s^{\prime}(E)-1\right]} \\
\text { where } s^{\prime}(E) & \left.=1+E \ln \left[\rho\left(E+\langle\Delta E\rangle_{\partial}\right) / \rho(E)\right) /<\Delta E\right\rangle_{d}
\end{aligned}
$$

The inversion temperature, which determines the temperature at which $\langle\Delta \mathrm{E}\rangle_{\mu}=0$ is nearly linearly dependent on the energy level of interest and is inversely proportional to the number of effective osciliators at the energy in question. For a given temperature the inversion energy of stable molecules is related to the average energy in the Boltzmann internal energy distribution. However, for unimolecular reaction systems, the situation is more complex.

The main difference in these formulations is that equation (9) is not energy dependent and cannot change sign; real molecules exhibit a sign change for $\langle\Delta E\rangle_{\text {sll }}$ as a function of both temperature and energy.

TR have also defined a variety of efficiency terms which are a function of temperature, stepsize, reactant and degree of falloff (or reaction order, $\Phi$ ). The TR formulation takes into consideration that the average energy of reacting molecules is a function of $\Phi$, while Troe's does not. This is important when one considers how the average energy of reacting molecules changes with both temperature and degree of falloff. The apparent activation energy for a reaction in the second order region can be substantially less then $E_{0}$. We have tested the validity of these equations for the recently reported unimolecular thermal decomposition of cyclohexene by Kiefer and Shah $^{27}(\mathrm{KS})$. This test was important since some conclusions as to the constancy of stepsize with temperature were made.

\section{Calculation of Vibrational-Energy Transfer Quantities at High Temperatures}

To compare the limitations of equations (9) and (10) we have used the cyclohexene system; for this system $E_{0}=61.9 \mathrm{kcal} / \mathrm{mol}$ and $T_{l}=1555 \mathrm{~K}$. Tablc $\mathrm{V}$ provides a summary of average energies along, with their defining equations. The inversion temperature is close to the middle of the reported experimental temptrature range and is where a deviation between equations (9) and (10) may possibly begin. (Of course, $T_{1}$ can increase and rise about $2000 \mathrm{~K}$ for relevant reaction energies in excess of $-90 \mathrm{kcal} / \mathrm{mol}$.)

We have calculated the relationship between $\langle\Delta E\rangle_{d \mu}$ and $\langle\Delta E\rangle_{d}$ for various energy parameters (defined below) and temperatures using the approximate equation (9) and improved equation (10) expressions. The results are exhibited in Figure 17 and Figure 18. The illustrative energy values chosen for the subject molecule are those that are relevant for the cyclohexene system. It is evident from these figures that the improved relationship is a strong function of the energy level. The approximate curve corresponds closely in shape to the more realistic curves at some fixed energy that varies with temperature.

\section{Energy level Specification for the Collision Process}

Actually, the specification of the relevant average energy level or range of energies for the collision process is not as clearly or easily estabilished for unimolecular systems as it is for stable molecules or for extemally energized reaction systems. The high-pressure regime is the only region for which the population distribution above $\mathrm{E}_{\mathrm{o}}$ is definitely known. (Paradoxically, it is for this condition that no information can be gained regarding energy transfer from these thermal unimolecular reactions.) As used widely, the representative energy at infinite pressure may be taken as the average energy of reacting molecules, $\langle E\rangle_{r p}$, given classically as $E_{o}+s R T$ [where $s$ is the effective number of oscillators not necessarily the same as s'(E) used in equation (11)]. The problem of energy level specification involves a particularly difficult deconvolution in the falloff region. Transport of molecules along the energy axis becomes an ever increasing aspect of the reaction mechanism as the pressure declines. This offers an element of complexity in the specification of relevant energy levels in the falloff region. Depletion of the higher energy populations below the Boltzmann equilibrium values occurs. KS have suggested that the representative energy range for their system, which was studied in the falloff region, did not go below $\mathrm{E}_{0}$. The implicit basis for their assertion is the fact that even in the limiting low-pressure region the average 
energy of reacting molecules only declines classically to the value $E_{0}+R T$ (for $E_{0} \gg s R T$ ). However, this proposition, which restrains the range of possible energy values, is incorrect.

For the zero pressure condition, the reaction process is purely molecular transport from the energy region below $E_{o}$, since molecules above $E_{o}$ do not experience collision and the relevant energy collision process involves only molecules below $E_{0}$. The possible extent of this energy range for weak colliders is suggested by the quantity $E_{1 m}$ used by TR to designate the energy level below $E_{0}$ at which the steady-state population of molecules converges to its equilibrium value. The weaker the collider the more complex and difficult the problem. For 'strong colliders' the excitation of molecules from levels below $\mathrm{E}_{\mathrm{o}}$ is dictated by the details of the strong collider probabilities. The situation above $\mathrm{E}_{\mathrm{o}}$ for weak colliders is particularly complex, as discussed previously. At a given degree of falloff, the form of the distribution of steady-state populations depends strongly on the assumed model for $\mathrm{P}\left(\mathrm{E}, \mathrm{E}^{\prime}\right)$. This difficulty is presently a bar to a detailed analysis that does not invoke excessive speculation regarding the true form of $P\left(E, E^{\prime}\right)$.

KS used equation (9) to correlate the values of $\langle\Delta \mathrm{E}\rangle_{\mu \mu}$ and $\langle\Delta \mathrm{E}\rangle_{\mathrm{d}}$. They argued that it appeared unlikely that a sign change in $\langle\Delta \mathrm{E}\rangle_{\mathrm{dl}}$ would be of importance under their conditions. In order to assess the practical discrepancy between equations (9) and (10), we have calculated these quantities for the exponential model. Equation (10) has been evaluated for representative energies stating at $E_{o}$ and encompassing all energies of reacting molecules at four representative temperatures; the extremes are shown in Figure 17 and Figure 18. Also shown in the plots are curves of the quantum statistical energies corresponding to the low and high pressure Tolman definition of a representative energy of the reacting molecules (Table V). For illustrative purposes indicial marks have been added which include the values of the energy of reacting molecules at pressures of 0.1 , 1 and 10 torr for a strong collider. These were arbitrarily chosen as known, easily calculable values in the range of interest. It is evident that below $2000 \mathrm{~K}$ the approximate relationship lies between the $p=0$ and $p=\infty$ curves of the improved correlation. At the highest temperature, $2000 \mathrm{~K}$, the approximate curve falls ouside this range. Moreover, it can also be seen from the curves in Figure 18 at $2000 \mathrm{~K}$ that inversion of $\langle\Delta \mathrm{E}\rangle_{\mu}$ occurs ai approximately $92 \mathrm{kcal} / \mathrm{mol}$, with increasingly positive values for $\mathrm{E}=80 \mathrm{kcal} / \mathrm{mol}$ and for $\mathrm{E}=\mathrm{E}_{0}=61.9 \mathrm{kcal} / \mathrm{mol}$. It is evident, therefore, that the two features discussed at the outset of this section, namely, specification of the average energy of the molecules undergoing collision is an important variable in the correlation between $\langle\Delta E\rangle_{d}$ and $\langle\Delta \mathrm{E}\rangle_{\mu \nu}$, and that at least at $2000 \mathrm{~K}$, for which the true order of reaction is highest, inversion effects become significant, i.e. equation (10) should be used.

If the Tolman definition for the average energy of the reaction molecules is not the relevant one, with the average, energy of colliding molecules being more appropriate, then at $2000 \mathrm{~K}$, the energy of reacting molecules at $\mathrm{p}=0$ would move below the inversion energy of $92 \mathrm{kcal} / \mathrm{mol}$, in fact below $E_{0}$. Indeed, in the low-pressurc region inversion would occur even at $1800 \mathrm{~K}$; some corrections would be of relevance also at $1500 \mathrm{~K}$.

Table VI presents calculated values for $\langle\Delta \mathrm{E}\rangle_{\mathrm{d}}$ as a function of temperature when $\langle\Delta \mathrm{E}\rangle_{\mathrm{d}}$ is held constant at $550 \mathrm{~cm}^{-1}$ and the values of $\langle\Delta \mathrm{E}\rangle_{\mathrm{d}}$ when $\langle\Delta \mathrm{E}\rangle_{\mathrm{dl}}$ is heid constant at $73 \mathrm{~cm}^{-1}$. These values were chosen since at $1500 \mathrm{~K}\langle\Delta \mathrm{E}\rangle_{\mathrm{d}}$ is $550 \mathrm{~cm}^{-1}$ and $\langle\text { de }\rangle_{\text {all }}$ is $-78 \mathrm{~cm}^{-1}$. A constant $\langle\Delta \mathrm{E}\rangle_{\text {dl }}$ requires $\langle\Delta \mathrm{E}\rangle_{\mathrm{d}}$ to increase with temperature; the increase is faster for equation (9) than it is for equation (10). Holding $\langle\Delta E\rangle_{d}$ constant requires that $-\langle\Delta \mathrm{E}\rangle_{\|}$decrease with an increase in temperature; equation (9) exhibits a more rapid change than equation (10).

The original conclusion of KS was that the constancy of $\langle\Delta \mathrm{E}\rangle_{\text {dll }}$ necessitated an increase in $\langle\Delta \mathrm{E}\rangle_{\mathrm{d}}$ with rise of temperature. This would indicate that a repulsive interaction model as contrasted to one of attraction was operative for energy transfer. The Landau-Teller model ${ }^{8}$ predicts an increase in $\langle\Delta E\rangle_{d}$ with rise in temperature. However, using the correct equations indicate that $\langle\Delta E\rangle_{d}$ remains constant at $\sim 500 \mathrm{~cm}^{-1}$. This is in accord with other reported results.

It is important when using generic equations that they be used in a tested frame, otherwise diagnostic calculations should be performed to test any limitations that may be involved. In practice the inversion temperature is a useful benchmark. The inversion temperature is a function of energy level so appropriate energies must be known; to a first approximation these energies can be calculated from the strong collision models. In spite of these simplifications, it is recommended that master equation calculations be performed for molecules with a high number of normal modes at high temperatures. High temperatures are those that exceed the inversion temperature at $E_{0}$. 


\section{REACTIONS STUDIED in which ENERGY TRANSFER viay be IMPORTANT}

The importance of energy transfer in directing the reactant to particular products was observed for two reactions: the infrared multiphoton excitation of perfluorocyclopropene and the uv photolysis 1,2-difluorodiiodoethene. A short summary of these experiments are presented.

\section{Perfluorcyclopropene: production of tetrafluoroallene and tetrafluoropropyne}

It was expected that the infrared multiphoton excitation (IRMPE) of tetrafluorocyclopropene (TFCP) would have two pathways: a ring opening producing a diradical or $\mathrm{CF}_{2}$ extrusion. The first pathway after 1,2 fluorine migration leads to tetrafluoroallene (TFA) and tetrafluoropropyne (TFP) while the second pathway directly produces tetrafluoroethylene; this product was not observed. Contrary to what is predicted for a simple unimolecular process, our IRMPE results show a dependence for the conversion per initial laser pulse on fluence and TFCP pressure while the product ratio is independent of these experimental parameters. No TFA or TFP was observed when an 8 torr sample of TFCP was heated to $250^{\circ} \mathrm{C}$; a high molecular weight product was observed. At this temperature TFA and TFP uniformly disappeared. Further details of these experiments and results have been published ${ }^{23}$.

The reactants and product were analyzed and identified by FT-IR spectroscopy. For initial TFCP pressures ranging from 0.4 to 8.5 torr, fluences from 0.8 to $2 \mathrm{~J}$, and percent conversions from 1 to $50 \%$, the TFA/TFP mole ratio for the 25 experiments was 1.0 and independent of the laser line used. The amounts of TFA and TFP formed were directly proportional to the loss of TFCP. The conversion per pulse increased markedly with an increase in initial TFCP pressure but decreased as the pressure of the products (i.e. \% reaction) increased. With unfocused radiation and an initial TFCP pressure of 0.2 torr there was no change in the spectrum after 1100 pulses, whereas with an initial pressure of 8 torr, 29\% of the TFCP was converted to TFA and TFP after a single $2 \mathrm{~J}$ pulse. The first laser pulse corresponds to about $100 \%$ corversion of the TFCP in the irradiated volume. The initial extent of reaction increases markedly with pressure; a 20 fold increase in pressure ( 0.4 to 8.0 torr) produces a factor of 100 in the initial conversion per pulse. No quantitative studies of the reaction at higher pressures were performed, but the conversion at 8.5 torr was lower than that at 8.0 torr.

With an initial pressure of 2 torr, 128 pulses of unfocused radiation resulted in 13\% conversion, but the same irradiation of a sample containing 1.5 torr of TFCP and 6.6 torr of argon produced no change in the TFCP spectrum. Argon efficiently removes the internal energy from TFCP while TFCP efficiently transfer its energy to another TFCP molecule (i.e V-V transfer is efficient for TFCP-TFCP collisions). In every case, the apparent isomerization reaction did not consume more than about $50 \%$ of the initial TFCP using the $931 \mathrm{~cm}^{-1}$ laser line; conversions up to $60 \%$ were observed when the $1083 \mathrm{~cm}^{-1}$ line was used. For example, 100 pulses of unfocused irradiation for an initial TFCP pressure of 8.1 torr resulted in $40 \%$ of the TFCP isomerized to TFA and TFP, 1000 additional pulses resulted in a total of $50 \%$ loss of TFCP, but there was also a $4 \%$ loss of TFA and a $10 \%$ loss of TFP relative to the amounts present after 100 pulses.

After isomerizing $42 \%$ of the TFCP in a sample with an initial pressure of 8.5 torr, the $\mathrm{CO}_{2}$ laser frequency was changed to $1039 \mathrm{~cm}^{-1}$ where TFA has a strong absorption band but TFCP doe not. After 66 pulses (1.3 $\mathrm{J} /$ pulse), no changes were observed in the infrared spectrum of the sample. Thus PFA is not efficient in transferring its vibrational energy to internal energy of TFCP required for reaction.

The dependence of the isomerization yield per laser pulse on the pressure of TFCP, the products, and/or a buffer gas (argon) suggests that the process is collision-assisted. The very high conversion per pulse at higher pressures and the inhibition of the reaction by argon or the products of the reaction would seem to rule out rotational relaxation or pressure broadening of the absorption line as sources of the observed pressure dependence. Intermolecular $\mathrm{V}-\mathrm{V}$ or $\mathrm{V}-\mathrm{T} / \mathrm{R}$ transfer as a result of collisions of excited TFCP with cold TFCP, TFA or TFP, or a buffer gas would be expected to cause a decrease in conversion per pulse with increasing pressure.

An energy pooling collision to yield one molecule with sufficient energy for ring opening might be invoked as an excitation mechanism; this would satisfy the observed pressure dependence. The observed insensitivity of the product ratio to fluence or pressure indicates that the process in not unimolecular so that this mechanism is less likely. However, by coincidence both unimolccular processes could have nearly identical critical energies and structures for the activated complex so that the results would then be consistent with energy pooling.

Another possible pathway which satisfies the pressure dependence and the PFA/PFP mole ratio would 
involve the collision of two TFCP molecules (either one or both supplying sufficient energy for reaction); during the collision a fluorine atom from each molecule is transferred to the other (requiring a large decrease in entropy). The vibrational energy content in either TFCP molecule would be less than the threshold required to form a diradical but sufficient to promote the transfer. The TFA/TFP ratio would be invariant with fluence and pressure and be equal to 1; however, the rate constant would show an energy dependence. This pathway would account for the difference between collisions of TFCP with argon, TFA, TFP and TFCP; the first three partners would remove the required vibrational energy from TFCP. Thus, as reaction proceeds, the initial excitation is collisionally transferred to TFA and TFP instead of being directed to the reaction coordinate as is the case for collision with TFCP. At this time there is not evidence to preclude this pathway, in fact the high conversion at low fluences, the pressure dependence and the PFA/PFP mole ratio are indicators that this may be the dominant path.

These studies have shown that laser excitation can be used to promote reactions with high critical energies in a low temperature environment so that consecutive reactions with smaller critical energies will be quenched. TFA and TFP appear to react stoichimetrically to produce some of the same products as the thermolysis of TFCP. Since the thermolysis of TFP does not produce TFA or the products resulting from the thermolysis of the TFCP-TFP-TFA mixture, it appears a cooperative process is involved for IRMPE.

\section{1,2-Difluorodiliodoethene: Production of difluoroacetylene}

Trans 1,2-difluorodiiodoethene ( $t$-DFDIE) was exposed to $248 \mathrm{~nm}$ radiation produced by a $\mathrm{KrF}$ excimer laser. The products: difluorcethyne(DFE), cis-DFDIE and 2,3-diiodo-1,2,3,4-tetrafluorocyclobutene(DITFCB) were identified and analyzed by FT-IR spectroscopy. The infrared spectrum of DFE was obtained at $0.03 \mathrm{~cm}^{-1}$ resolution.

Recently, vibrational constants have been calculated for $\mathrm{DFE}^{29}$; the preparation of and characterization of DFE has been difficult ${ }^{30,31}$. Our experiments and DFE precursor are different than those recently reported which involved photodissociation of difluoromaleic anhydride in an argon matrix ${ }^{30}$ or the thermolysis of perfluoro-1,2,3-triazine ${ }^{31}$. An indicator of the reactivity of DFE is its half-life in our system: 30 minutes in a glass cell and less than 15 minutes in an aluminum cell; a complication when high resolution spectra are accumulated.

The results presented here are not complete hut indicate some of the complexity of the reaction in this system and the importance of energy transfer in producing unstable products. Experiments were performed in the 0.5 to 1.0 torr pressure region with pulse energies between 20 to 110 mjoule and exposures ranging from 10 to 1000 pulses. The DFE yield appears to be optimum with smaller pulse energy and lower pressure of DFDIE; the yield first increases with the initial number of pulses and then decreases with further exposure time. The yield of cis-DFDIE increases with exposure as does the yield of DITFCB.

Elementary steps which make up a mechanism which fits these observations are

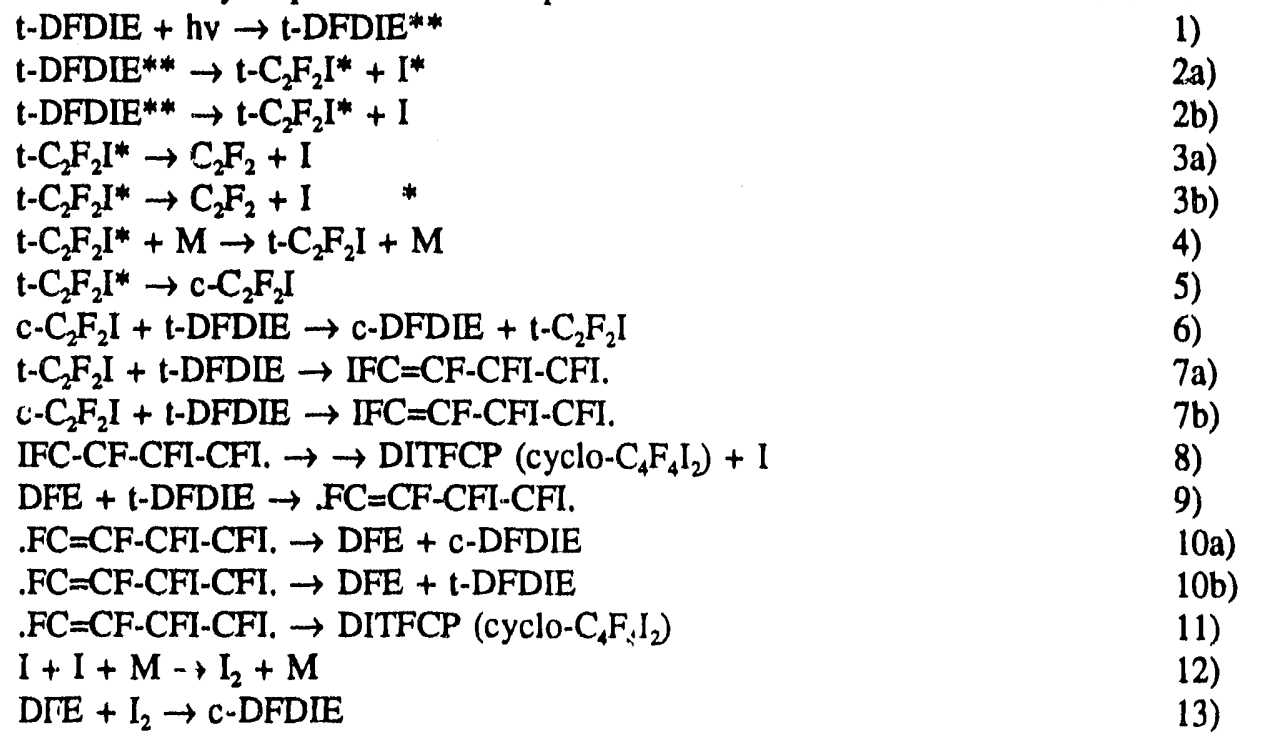


The primary photon provides $115 \mathrm{kcal} /$ mole to DFDIE; the C-I bond dissociation energy is approximately 53 $\mathrm{kcal} / \mathrm{mole}$. The excess energy of $62 \mathrm{kcal} / \mathrm{mole}$ after dissociation can be distributed between the internal modes of the $\mathrm{t}-\mathrm{C}_{2} \mathrm{~F}_{2} \mathrm{I}$ radical, electronic excitation of $\mathrm{I}(21.7 \mathrm{kcal} / \mathrm{mole})$ and relative translation. For an analogous molecule, Lee and coworkers ${ }^{32}$ have observed for the photodissociation of $\mathrm{C}_{2} \mathrm{~F}_{\mathrm{g}} \mathrm{I}$ that $\mathrm{I}^{*}$ is the predominant channel (2a) and approximately $21 \mathrm{kcal} / \mathrm{mole}$ is partitioned into relative translational energy. Thus it is estimated that the $\mathrm{t}-\mathrm{C}_{2} \mathrm{~F}_{2} \mathrm{I}$ radical has approximately $20 \mathrm{kcal} / \mathrm{mole}$ of internal energy. Due to the formation of the pi bond in DFE, the $20 \mathrm{kcal} /$ mole should be sufficient to promote the sequential unimolecular reaction producing DFE via (3a) [(3b would be endoergic]; collisional energy transfer (4) can stabilize the radical so that reaction (3a) is suppressed. The unknowns for this scheme are the energy partitioning of the initial photodissociation and the critical energy for reaction (3). The observation that cis-DFDIE and DFDICB increase linearly with exposure indicates that these products are formed from a common primary precursor, i.e. reactions 4-8 and/or 9-11. In the latter, a unimolecular competition between dissociation and ring closure exists; the branching will be energy dependent and thus determined by energy transfer efficiencies. The decrease of DFE with exposure may indicate photon absorption by DFE followed by reaction with t-DFDIF (9-11) or with $I_{2}(13)$. These reactions are consistent with the observations that DFE yield is greatest for low DFDIE pressure and laser intensity. For these conditions, radical concentrations are low and energy stabilization (4) is decreased so that the unimolecular step

(3) becomes important.

The high resolution FT-IR spectrum in the 1330 to $1360 \mathrm{~cm}^{-1}$ (C-F stretch) exhibited a complex set of rotational progressions. In some subregions a 3:1 intensity variation appeared while in others a more chaotic line distribution was observed. The alternations are expected since DFE obeys Fermi-Dirac statistics; the intensity attributed to a specific transition is determined by the symmetry of the nuclear, rotational and vibrational states. In the case of $\mathrm{C}_{2} \mathrm{H}_{2}$, the spectral region for the $\mathrm{C}-\mathrm{H}$ stretch exhibits a clean and predicted 3:1 intensity variation. In the case of $\mathrm{C}_{2} \mathrm{~F}_{2}$ there are 2 bending modes with vibrational frequencies of 258 and $282 \mathrm{~cm}^{-1}$ so that room temperature hot bands are observed; approximately $46 \%$ of DFE is in the zero point energy state. As a consequence the C-F absorption is composed of a variety of transitions. The vibrational couplings and moments of inertia will be different for these states so that the resulting absorptions are shifted. This complication of overlapping hot bands prevented us from assigning a band origin for the ground state transition. 


\section{SUMMARY}

This report describes completed work relating to collisional energy transfer. We have studied some of the parameters which influence energy transfer via experiments and computer calculations; developed experimenta! methodologies and analysis schemes; determined the effect of energy transfer on high temperature unimolecular systems and assessed the limitations of commonly used approximations; and we have observed new chemical reactions in which energy transfer exhibited specific behavior.

Intermolecular energy transfer of highly excited polyatomic molecules plays an important role in many complex chemical systems: combustion, high temperature and atmospheric chemistry. We have designed and constructed equipment which monitors the relaxation of vibrational energy for polyatomic molecules; computerized data acquisition and processing were necessary for these experiments. A phase sensitive Mach Zehnder interferometer was built and tested: preliminary results indicate that this will be a fruitful technique for obtaining energy transfer information.

Trepds in the collisional efficiency $(\beta)$ for energy transfer as a intion of the substrate's excitation energy and the complexities of substrate and deactivator were observed. For a given substrate $\beta$ increases as the deactivator's mass increases to $\sim 30$ amu and then exhibits a nearly constant value; these results are interpreted in terms of a mass misinatch between the atoms of the colliders. In a homologous series of fluorinated substrate molecules $\left(C_{3}-C_{8}\right) \beta$ decreases as the number of carbon atoms in the substrate increases; replacing $F$ with $H$ increases $\beta$. All substrates, except for $\mathrm{CF}_{2} \mathrm{Cl}_{2}$ and $\mathrm{CF}_{2} \mathrm{HCl}$ below $10,000 \mathrm{~cm}^{-1}$, exhibited the independence of $\beta$ and energy so that $\langle\Delta E\rangle_{\mu \mu}$ is linear with energy. The res'ilts are interpreted by assuming that there is a statistical distribution of the intemal energy and the relaxation is controlled by a single oscillator; $\beta$ becomes a function of the energy in the oscillator and the lowest vibrational frequency of the substrate.

The application of energy transfer to high temperature unimolecular reactions was also studied: these limitations include the steady state correction and weak collider effects. Current approximations were evaluated and better approximations were developed. The importance of energy transfer in product yields was also experimentally observed in the photoisomerization of perfluorocyclopropene which exhibits specificity for this transfer of energy from substrate to deactivator. Because of inergy transfer rates we were able to produce difluoroethyne from the photodecomposition of diiodoe:hene: this is a new technique for producing this ill/usive compound.

During the duration of this project there was one postdoctoral associate (1 person-year), three graduate students ( 1 domestic and 2 international students: 4 people-years) and the PI that worked on this project. During the project all three graduate students switched to other research advisors; the overall project was beyond their capabilities (no advanced degrees were received). In spite of the insufficient amount of competent labor the proposed work was performed. The following papers were presented by the PI (except for \#3):

1. Monitoring Vibrational Relaxation of Highly Excited Molecules: Phase Sensitive Interferometry at the Midwest ACS meeting, November 6-8, 1991

2. Vibrational Relaxation of Highly Excited Pentatomic Molecules: $\mathrm{CF}_{2} \mathrm{HCl}$ and $\mathrm{CF}_{2} \mathrm{Cl}_{2}$ at the Midwest ACS meeting, November 7-9, 1990

3. V-T Energy Relaxation from Highly Excited Polyatomic Molecules: Fluotinated Hydrocarbons $\left(C_{3}-C_{8}\right)$ at the Midwest ACS meeting, November 1-3, 1989 [poster presented by B.H. Song]

4. The Importance of Weak Collisions on High Temperature Unimolecular Reactions in the Falloff at the Second International Conference on Chemical Kinetics, July 24-27, 1989 [poster] 5. V-T Relaxation of Vibrationally Excited Polyatomics: The Series $\mathrm{C}_{n} \mathrm{~F}_{2 n 2}(3 \leq n \leq 8)$ at the Gordon Conference on Molecular Energy Transfer, July 9-14, 1989 [poster]

6. Determination of Stepsize Parameters for Intermolecular Vibrational Energy Transfer at the Eleventh Combustion Research Conference, June 7-9, 1989

7. Determining Energy Transfer Step Size Quantities for High Temperature Unimolecular Reactions, Midwest ACS meeting, November 16-18, 1988

8. A 1,2-Fluorine Migration in the Multiphoton Isomerizatin of Tetrafluorocyclopropene at Lasers '87 in December 1987

The following publications resulted from work related to this project: 
"Infrared Multiphoton-Induced Isomerization of Tetrafluorocyclopropene", H.B. Friedrich, D.J. Burton and D.C. Tardy, J. Phys. Chem. 91, 6334 (1987)

"A 1,2-Fluorine Migration in the Multiphoton Isomerization of Tetraflnorocyclcpropene", D.C. Tardy, H.B. Friedrich and D.J. Burton, Proceedings for Conference on Lasers, 1987, STS Press, McLean, Virginia

"High Temperature Deviations from RRKM Unimolecular Rate Coefficients Expressions", I. Oref and D.C. Tardy, J. Chem. Phys. 91, 205 (1989)

"Calculation of Vibrational Energy Transfer Quantities: Temperature and Energy Dependence", D.C. Tardy and B.S. Rabinovitch, J. Phys. chem. 94, 1876 (1990)

"Energy Transfer in Highly Excited Large Polyatomic Molecules", I. Oref and D.C. Tandy, Chem. Rev. 90, 1407 (1990)

"Reply to Comment on 'High Temperature Deviations from RRKM Unimolecular Rate

Coefficients Expressions'", I. Oref and D.C. Tardy, J. Chem. Phys. 9A, 830 (1991) 


\section{APPENDIX}

\section{References}

1. I. Oref and D.C. Tardy, Chem. Rev. 90,1407 (1990)

2. R.T. Bailey, F.R. Cruickshank, R. Guthrie, D. Pugh, I.J.M. Weir, Molec. Phys. 48, 81 (1983)

3. K.M. Beck, A. Ringwelski, R.J. Gordon, Chem. Phys. Lett. 121, 529 (1985)

4. K.M. Beck and R.J. Gordon, J. Chem. Phys. 89, 5560 (1988)

5. W. Braun, I. Price, B.C. Cadoff, N.C. Peterson, Int. J. Chem. Kinet. 21, 1029 (1989)

6. R. Sobczynski, D.W. Setser and A.r. Slagle, J. Chem. Phys. 92, 1132 (1990)

7. J.D. Lambert Vibrational and Rotational Relaxation in Gases, Clarendon Press, Oxford, U.K. 1977

8. J.T. Yardley, Introduction to Molecular Energy Transfer, Academic Press, New York, 1980

9. R.N. Schwartz, Z.I. Slawsky, K.F. Herzfeld, J. Chem. Phys. 20, 5091 (1952)

10. R.D. Levine and R.B. Bernstein, Molecular Reaction Dynamics and Chemical Reactivity, Oxford University Piess, New York, 1987

11. C.C. Davis, Phys. Lett. 36, 515 (1980)

12. F.R. Grabiner, D.R. Siebert, G.W. Flynn, Chem. Phys. Lett. 17, 189 (1972)

13. a) J.M. Zellweger, T.C. Brown and J.R. Barker, J. Phys. Chem 90, 461 (1986). b) J. Shi and J.R. Barker, J. Chem. Phys. 88, 6219 (1988), c) M.L. Yerram, J.d. Brenner, K.D. King and J.R. Barker, J. Phys. Chem. 94, 6341 (1990), c) B.M. Toselli, J.D. Brenner, M.L. Yerram, W.E. Chin, K.D. King and J.R. Barker, J. Chem. Phys. 95, 176 (1991)

14. J.F. Durana and J.D. McDonald, J. Chem. Phys. 64, 2518 (1976)

15. W.L. Hase, R.J. Duchovic, D. Lu, K.N. Swamy, R.V.S. Linde, R.J. Wolf, VENUS: A General Monte Carlo Classical Trajectory Computer Program, ?uantum Chemistry Exchange Program, Indiana University, Bloomington, Indiana, 1989

16. A.R. Whyte, K.F. Lim, R.G. Gilbert and W.L. Hase, Chem. Phys. Lett. 152, 377 (1988)

17. W.H. Miller, W.L. Hase and C.L. Darling J. Chem. Phys 91, 2863 (1989)

18. J.M. Bowman, B. Gazdy and Q. Sun, J. Chem. Phys. 91, 2859 (1989)

19. I. Oref and D.C. Tardy, J. Chem. Phys. 91, 205 (1989)

20. D.C. Tardy and B.S. Rabinovitch, J. Phys. Chem. 94, 1876 (1990)

21. E.W. Montroll and K.E. Shuler, Adv. Chem. Phys. 1, 361 (1958)

22. S. Nordholm and H.W. Schranz, Chem. Phys. 62, 459 (1981) 
23. J. Troe, J. Chem. Phys. 66, 4745, 4758 (1977)

24. D.C. Tardy and B.S. Rabinovitch, Chem. Rev. 77, 369 (1977)

25. D.C. Tardy and B.S. Rabinovitch, J. Phys. Chem. 89, 2442 (1985)

26. D.C. Tardy and B.S. Rabinovitch, J. Phys. Chem. 90, 118\% (1986)

27. J.H. Kiefer and J.N. Shah, J. Phys. Chem. 91, 3024 ,1987)

28. H.B. Friedrich, D.J. Burton and D.C. Tardy, J. Phys. Chem. 91, 6334 (1987)

29. M.M Gallo and H.F. Schaefer, J. Chem. Phys. 93, 865 (1990)

30. J.C. Brahms and W.P. Dailey, J. Am. Chem. Soc. 111, 8940 (1989)

31. H. Burger, W. Sommer, J. Chem. Soc. Chem. Comm. 456 (1991)

32. D. Krajnovich, L.J. Butler and Y.T. Lee, J. Chem. Phys. 81, 3031 (1984) 


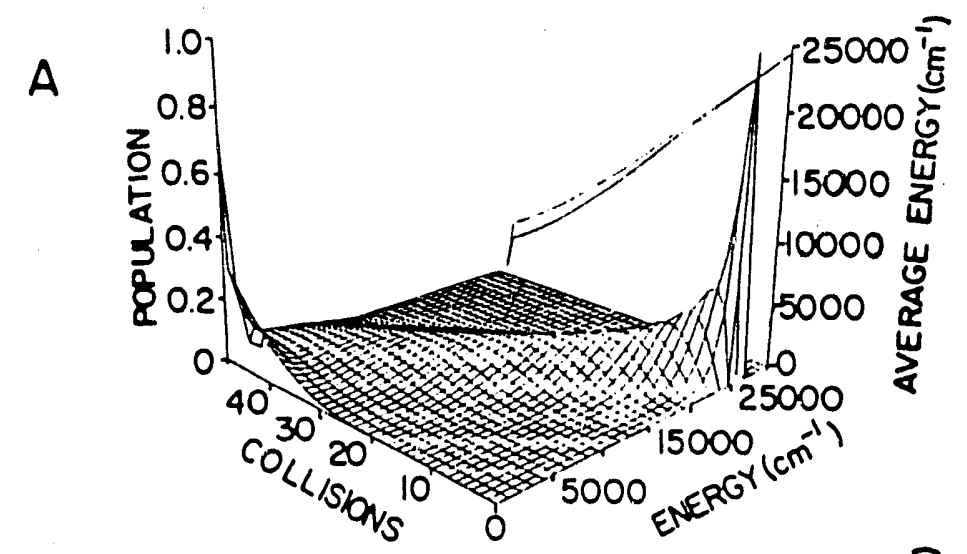

$B$
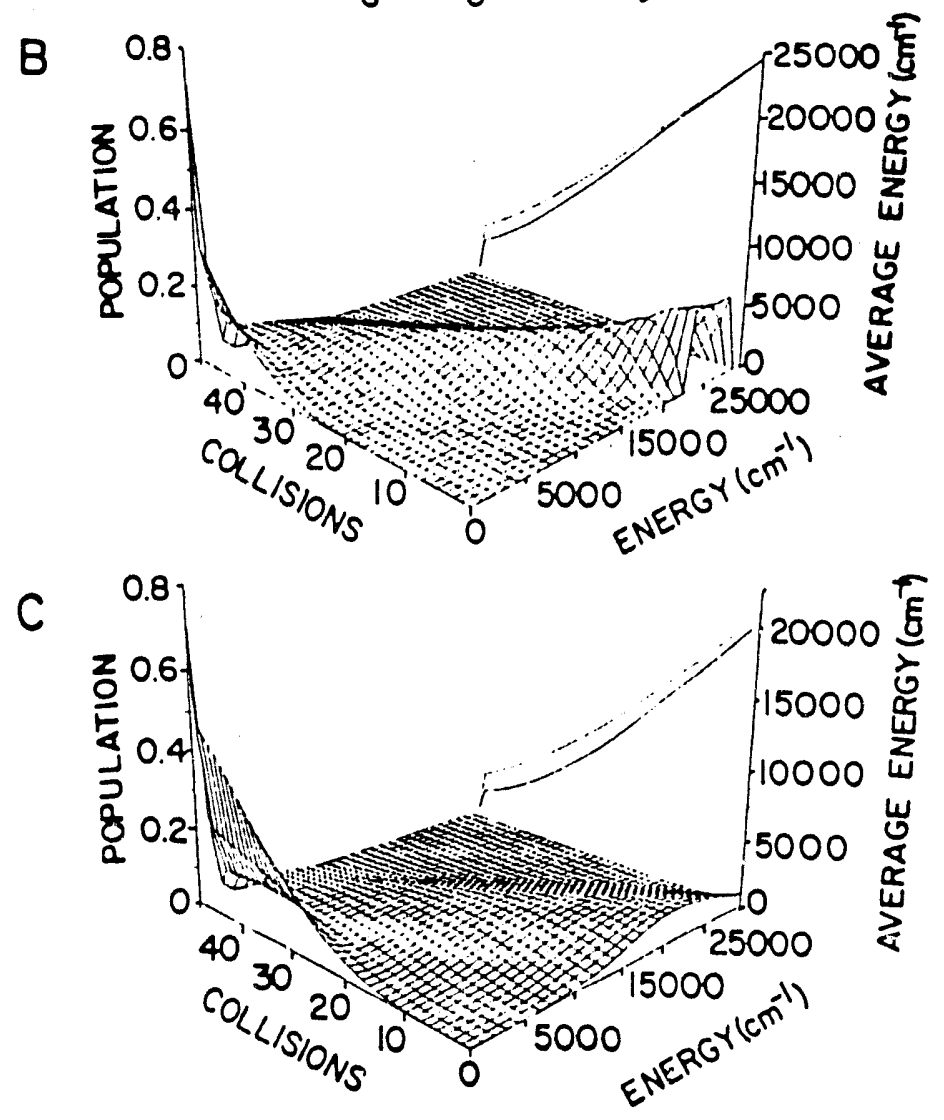

Fig 1. Relaxation of cyclohexene with exponential weak collider model. Population as a function of energy and number of collisions. The average energy, $<E>$ (solid line) and $\left\langle E^{2}\right\rangle^{0.5}$ (dashed line) are included.
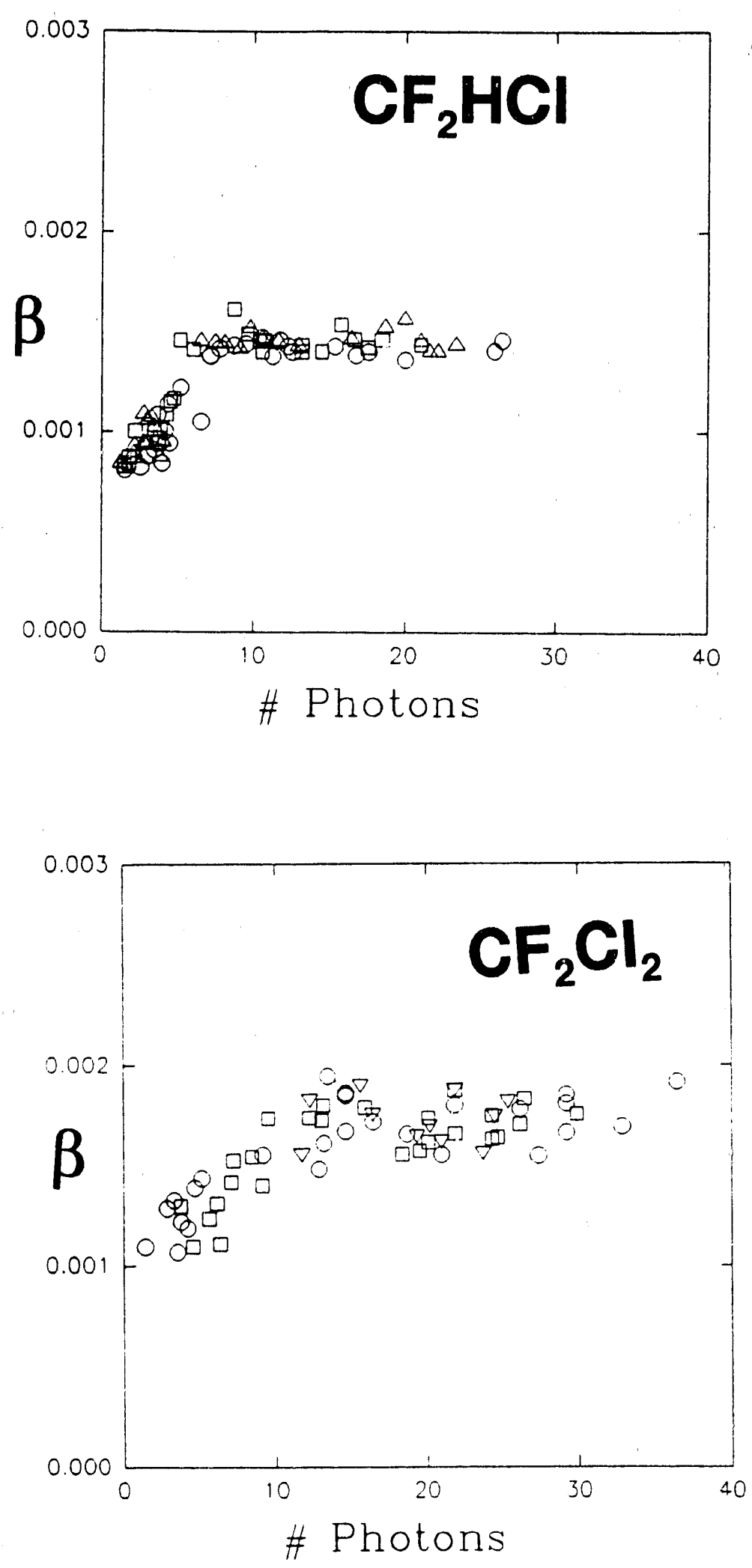

Fig 2. Collision efficiency, $\beta$, vs \# photons $\left(1000 \mathrm{~cm}^{-1}\right.$ per photon) for $\mathrm{CF}_{2} \mathrm{Cl}_{2}$ and $\mathrm{CF}_{2} \mathrm{HCl}$ systems with: $\mathrm{He}(\nabla), \mathrm{Ne}(\square), \operatorname{Ar}(0)$ and $\mathrm{Kr}(\Delta)$ 


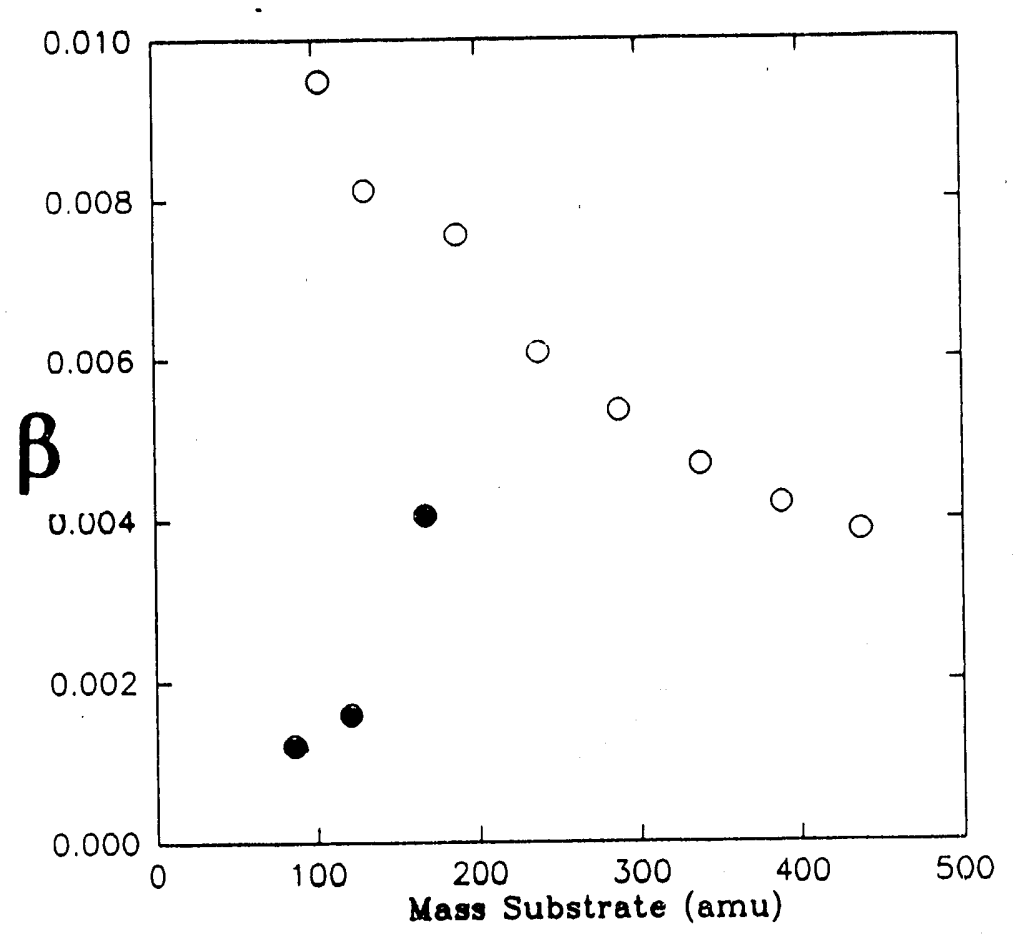

Fig 3. Collision efficiency, $\beta$, for argon vs mass of substrate. Open circles in order of increasing substrate mass: $\mathrm{C}_{6} \mathrm{H}_{13} \mathrm{~F}, \mathrm{C}_{8} \mathrm{H}_{17} \mathrm{~F}, \mathrm{C}_{3} \mathrm{~F}_{8}, \mathrm{C}_{4} \mathrm{~F}_{10}, \mathrm{C}_{5} \mathrm{~F}_{12}, \mathrm{C}_{6} \mathrm{~F}_{14}$, $\mathrm{C}_{7} \mathrm{~F}_{16}, \mathrm{C}_{8} \mathrm{~F}_{18}$. Filled circles in order of increasing mass: $\mathrm{CF}_{2} \mathrm{HCl}, \mathrm{CF}_{2} \mathrm{Cl}_{2}, \mathrm{C}_{6} \mathrm{~F}_{5} \mathrm{H}$.

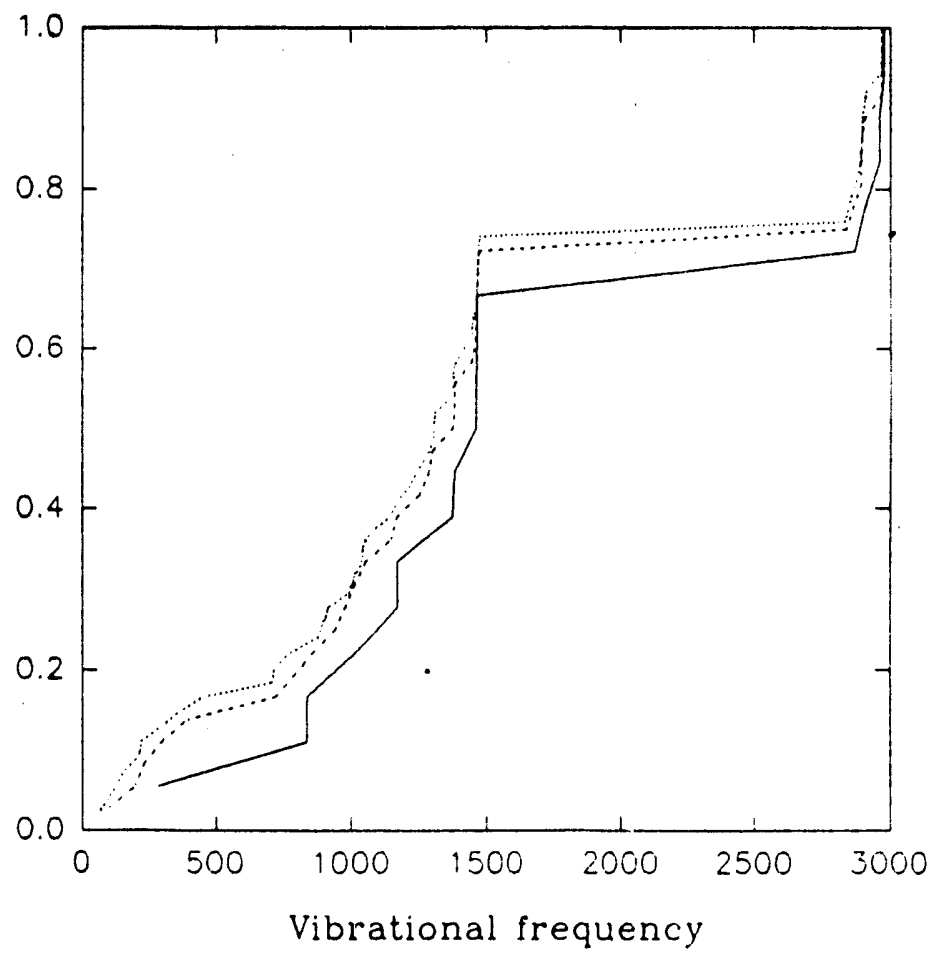

Fig. 4. Cumulative fraction of all vibrational modes vs vibrational frequency for: $\mathrm{C}_{2} \mathrm{H}_{6}$ (solid line), $\mathrm{C}_{4} \mathrm{H}_{10}$ (dashed line), $\mathrm{C}_{6} \mathrm{H}_{14}$ (short dashed line) and $\mathrm{C}_{8} \mathrm{H}_{18}$ (dotted line). 


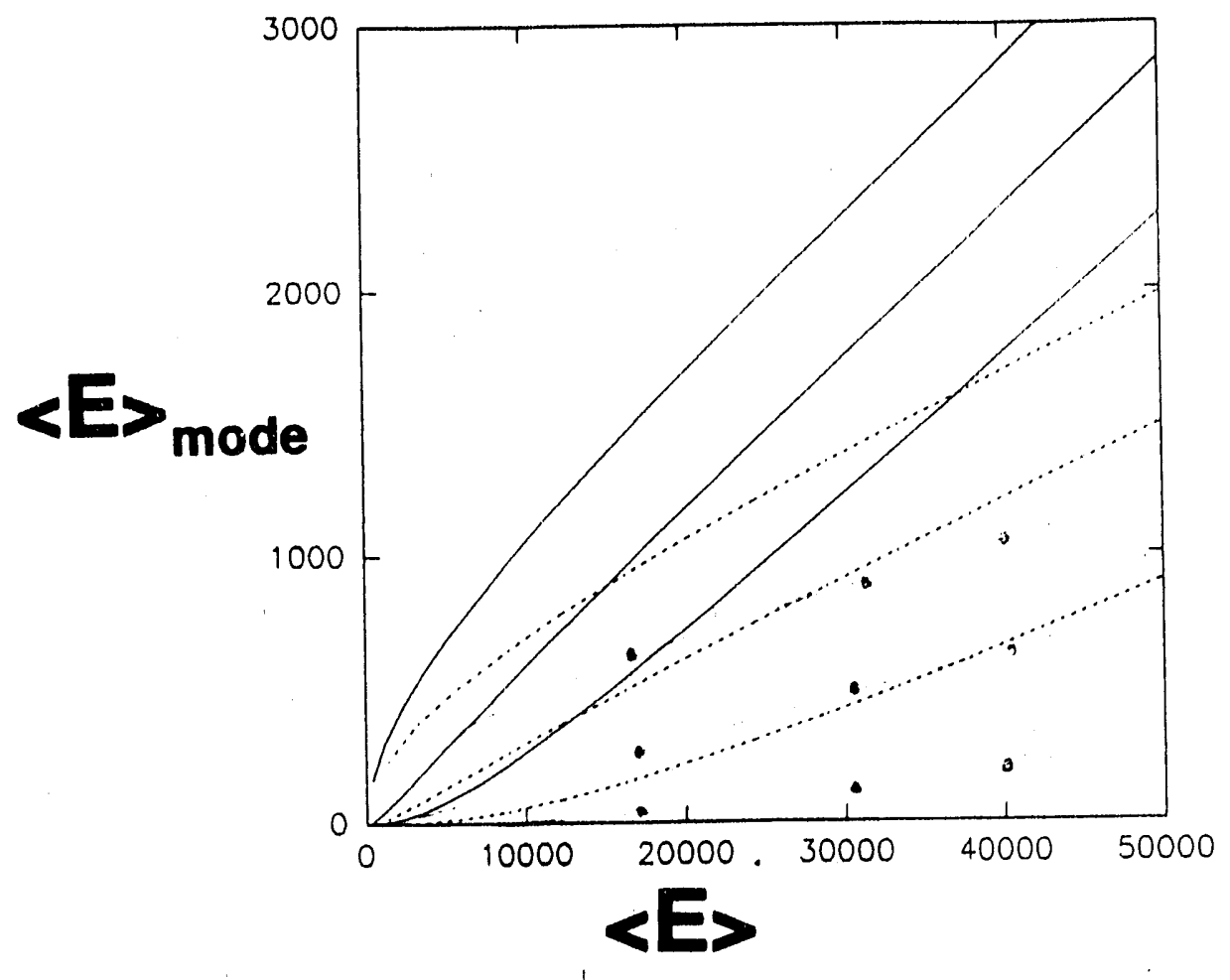

Fig. 5. Average energy in a vibrational mode, $\langle E\rangle_{\text {mode, }}$, vs total average energy for $\mathrm{C}_{2} \mathrm{H}_{6}$ (solid line), $\mathrm{C}_{4} \mathrm{H}_{10}$ (dashed line) and $\mathrm{C}_{8} \mathrm{H}_{18}$ (dotted line). The three curves for-each molecule represents the minimum (upper curve), mean and maximum (lower curve) vibrational frequency. For $\mathrm{C}_{2} \mathrm{H}_{6}, \mathrm{C}_{4} \mathrm{H}_{10}, \mathrm{C}_{8} \mathrm{H}_{18}$ these frequencies $\mathrm{cm}^{-1}$ are: $(279,1504,2977),(102,1205,2969)$ and $(37,1030,2968)$, respectively.

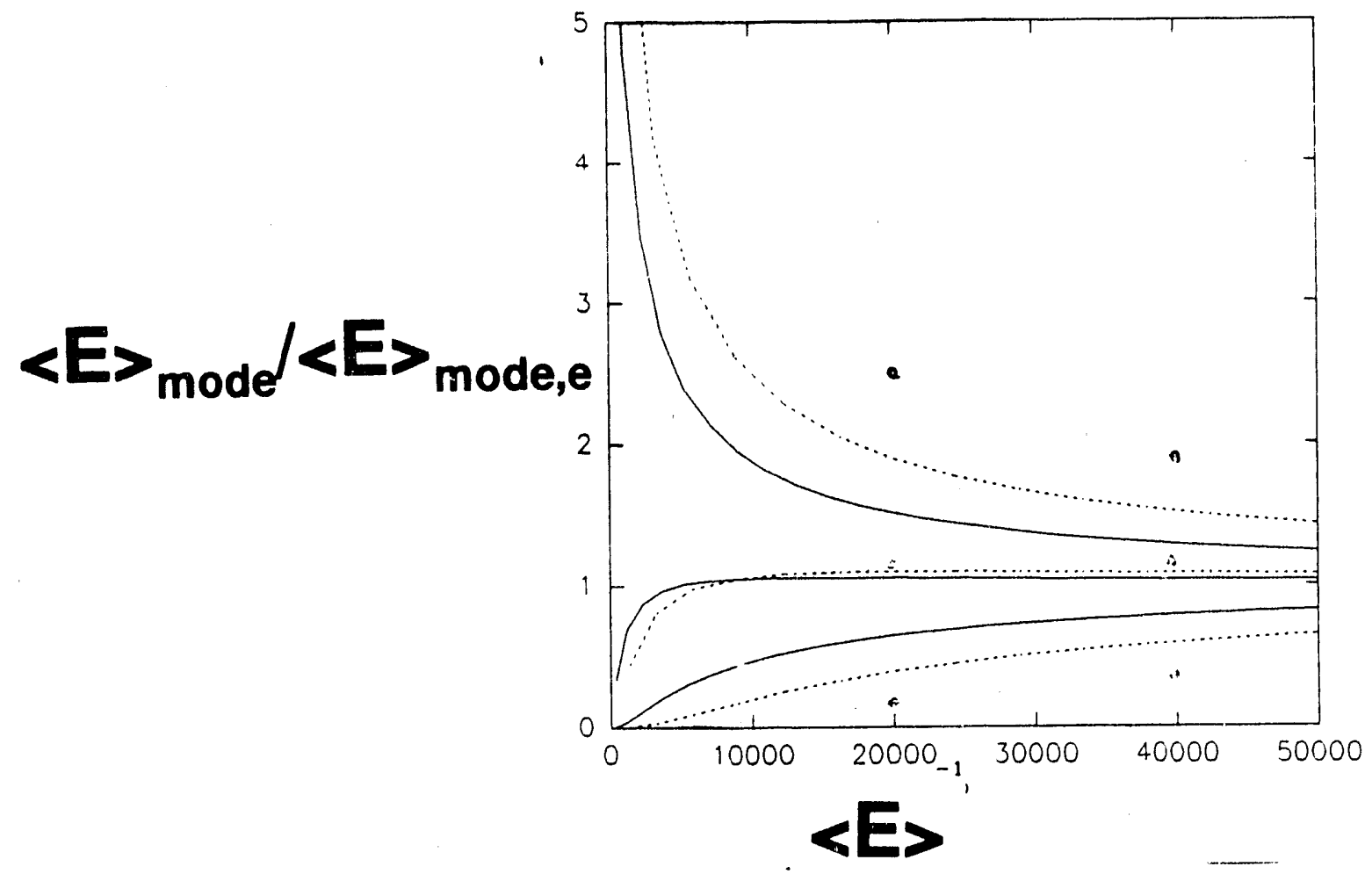

Fig. 6. Average energy in a vibrational mode, $\langle E\rangle_{\text {mode }}$, divided by the energy, $\langle E\rangle_{\text {mode.e }}$ if the total average energy was equally distributed vs total average energy. The legend of the curves is the same as that given in Fig. 5 . If the energy was equally distributed all points would be set to unity. 


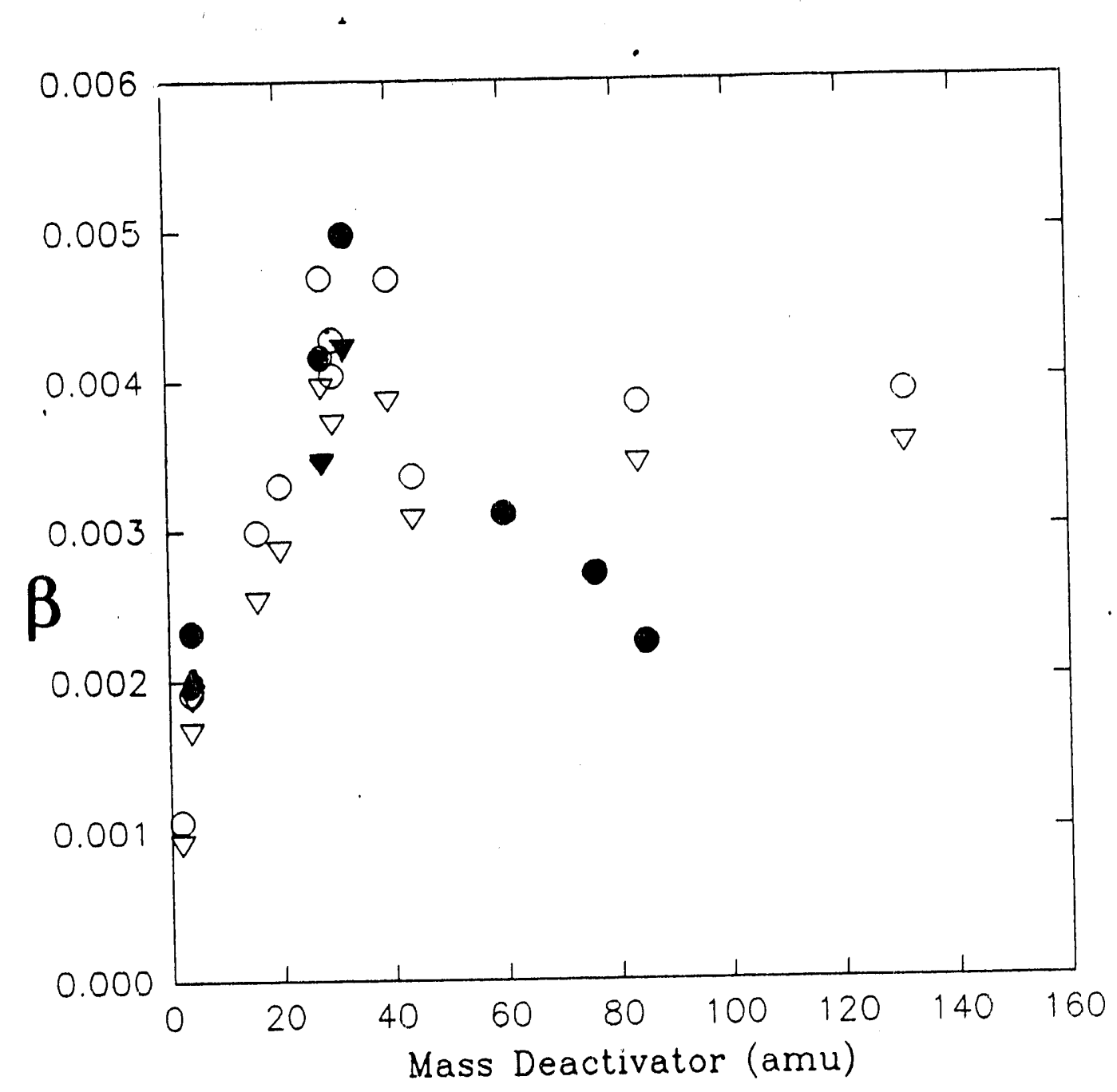

Fig. 7. Collision efficiency, $\beta$, vs mass of deactivator for $C_{6} F_{14}(0)$ and $C_{8} F_{18}(\nabla)$ substrates. Open symbols in order of increasing deactivator mass: $\mathrm{H}_{2}, \mathrm{D}_{2}, \mathrm{CH}_{4}, \mathrm{Ne}, \mathrm{CO}, \mathrm{C}_{2} \mathrm{H}_{6}, \mathrm{O}_{2}$, $\mathrm{CO}_{2}, \mathrm{Kr}, \mathrm{Xe}$. Filled symbols in order of increasing deactivator mass: $\mathrm{He}, \mathrm{N}, \mathrm{NO}, \mathrm{COS}$, $\mathrm{CS}_{2}, \mathrm{CH}_{2} \mathrm{Cl}_{2}$. 


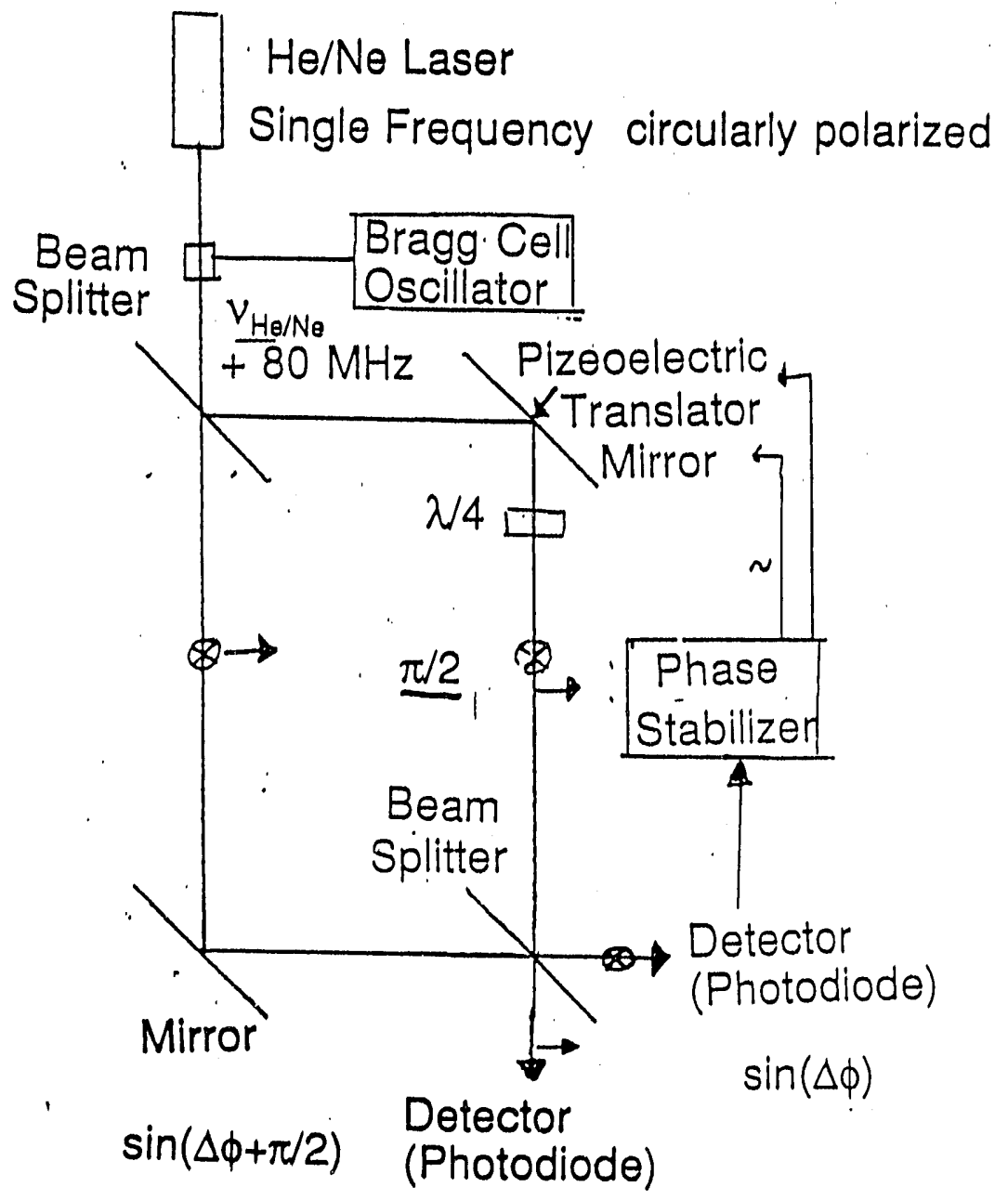

Fig. 8. Stabilized Mach Zehnder interferometer using two planes of polarization.

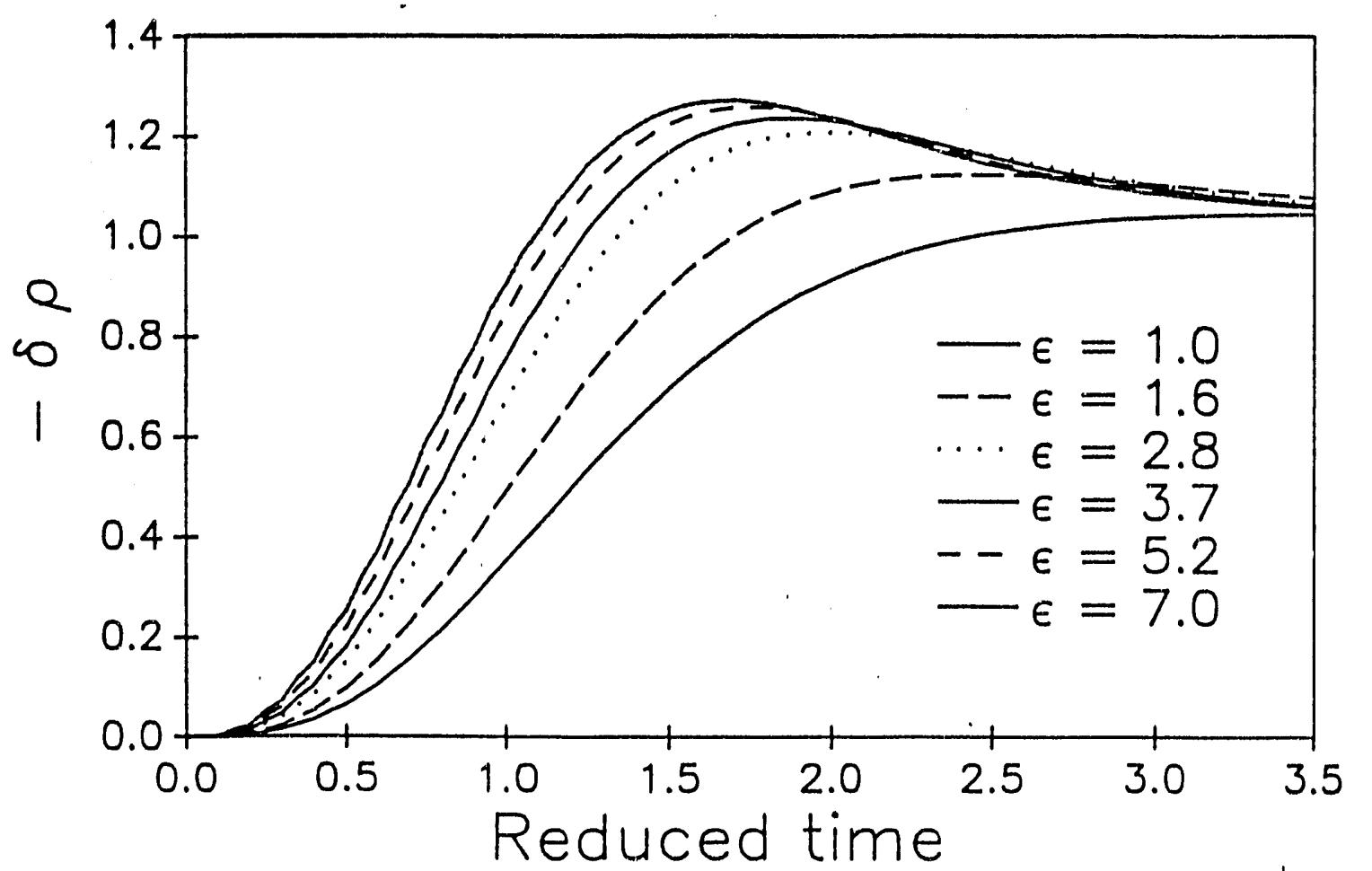

Fig. 9. Calculated plots of the change in gas density vs reduced time $\left(t / \tau_{s}\right)$ for various values of $\varepsilon\left(\tau_{V T} / \tau_{s}\right)$ where $\tau_{s}$ is the time for the acoustic wave to traverse the radius of the probe laser and $\tau_{\mathrm{VT}}$ is the time for $\mathrm{V}-\mathrm{T}$ relaxation. 


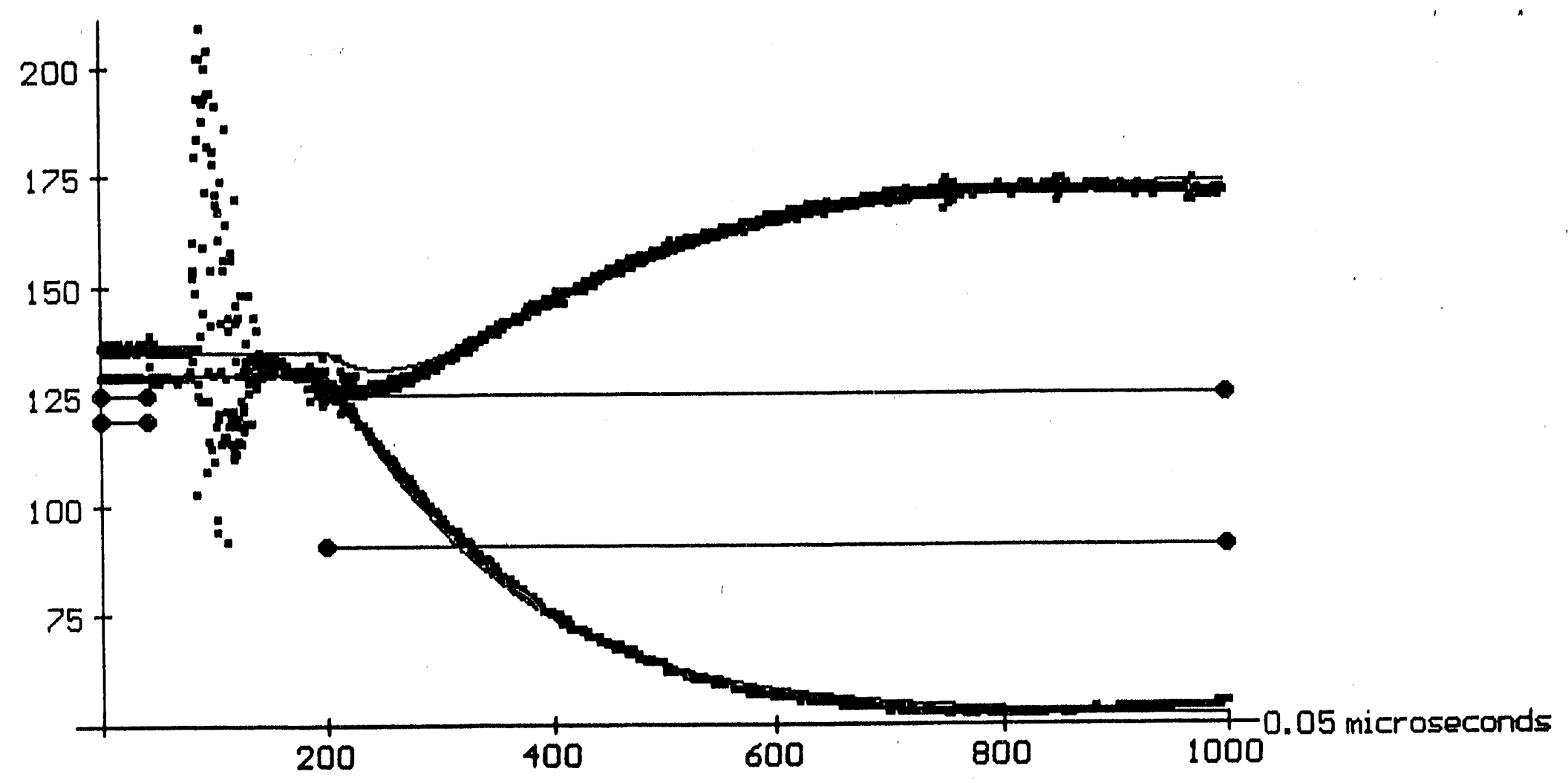

Fig 10. Observed quadrature signals from Mach Zehnder interferometer for 8 torr of $\mathrm{C}_{2} \mathrm{H}_{4}$. The time between each experimental point is 50 nanoseconds. The horizontal lines correspond to the times that were used for the least squares parameter optimization. Except for the points from the laser noise, the best fit line is nearly coincident with the experimental points.

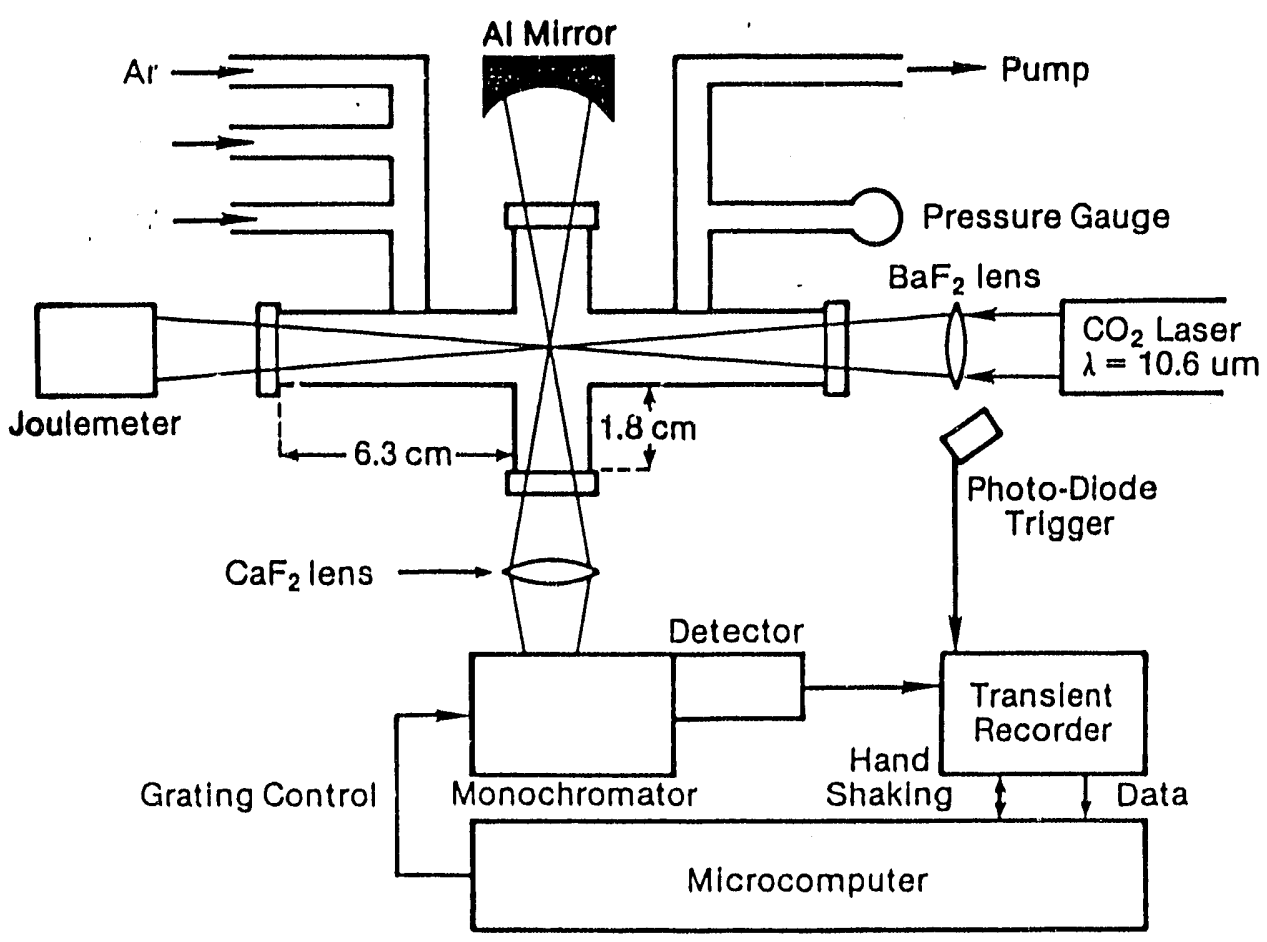

Fig. 11. Schematic of apparatus used for infrared fluorescence experiments. 


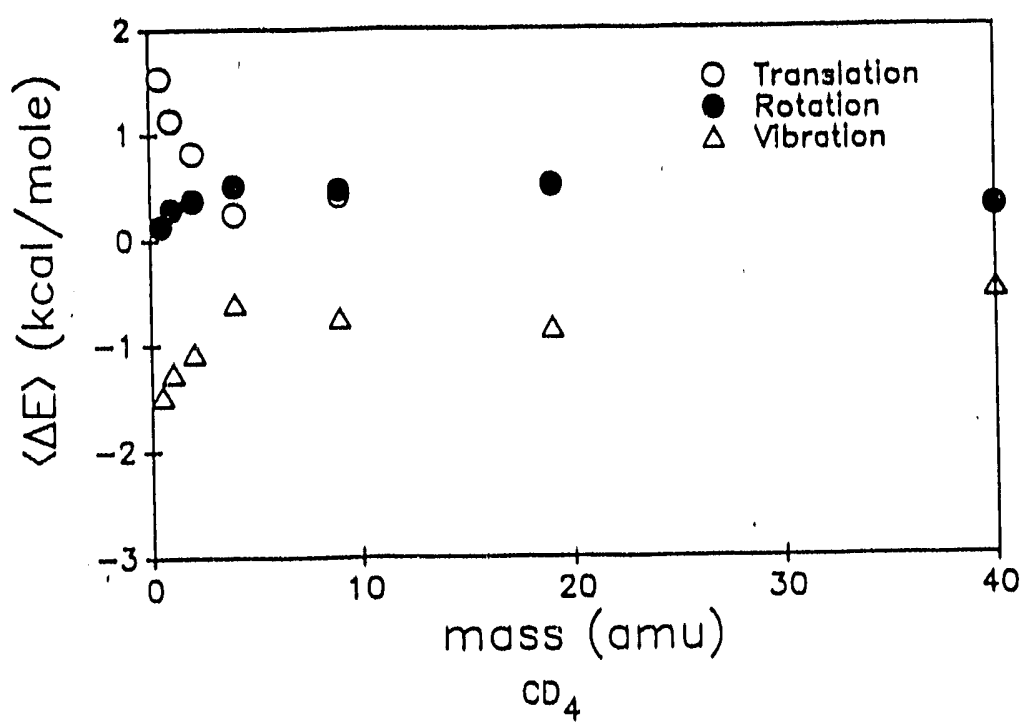

$\mathrm{CH}_{4}$

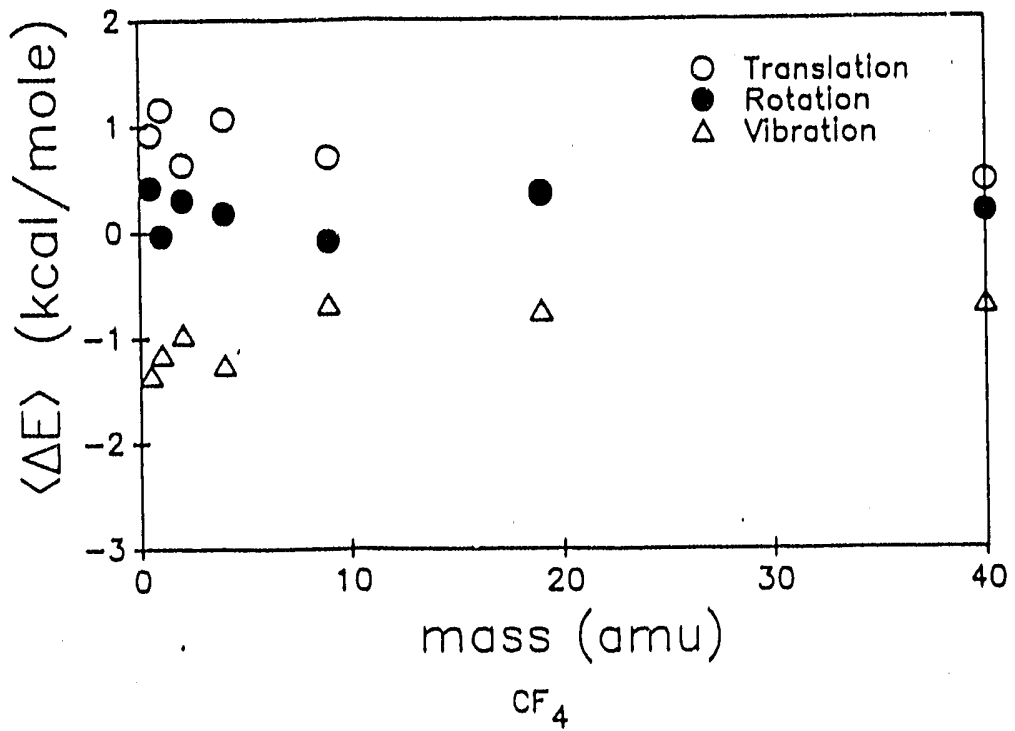

$\mathrm{CD}_{4}$

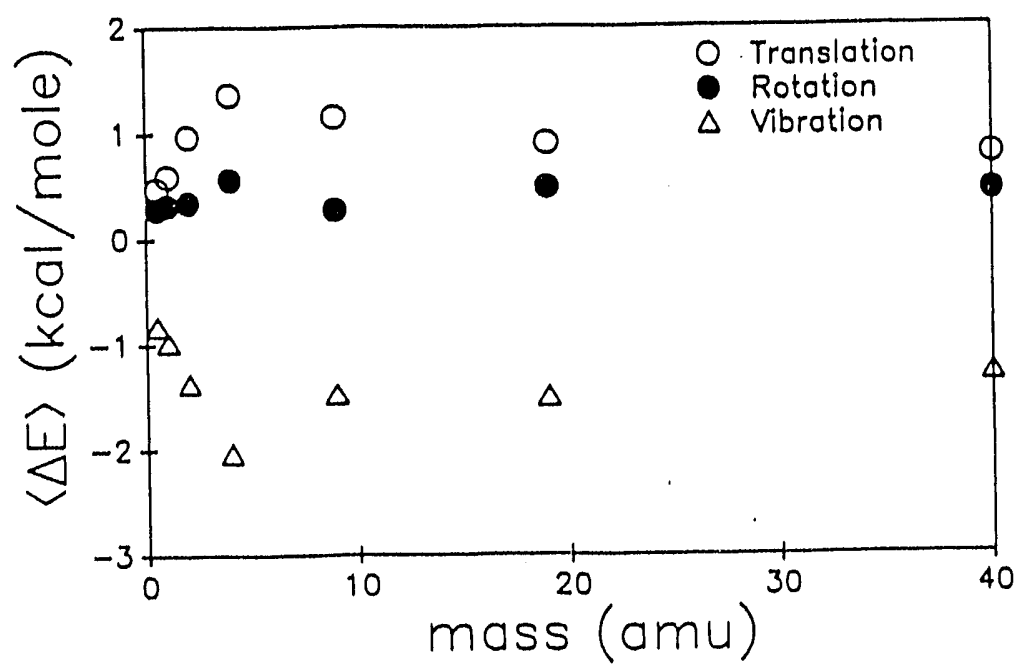

$\mathrm{CF}_{4}$

Fig. 12. Plots of $\langle\Delta E\rangle_{\text {all }}$ vs mass of deactivator for $C H_{4}, C D_{4}$ and $C F_{4}$ systems. 

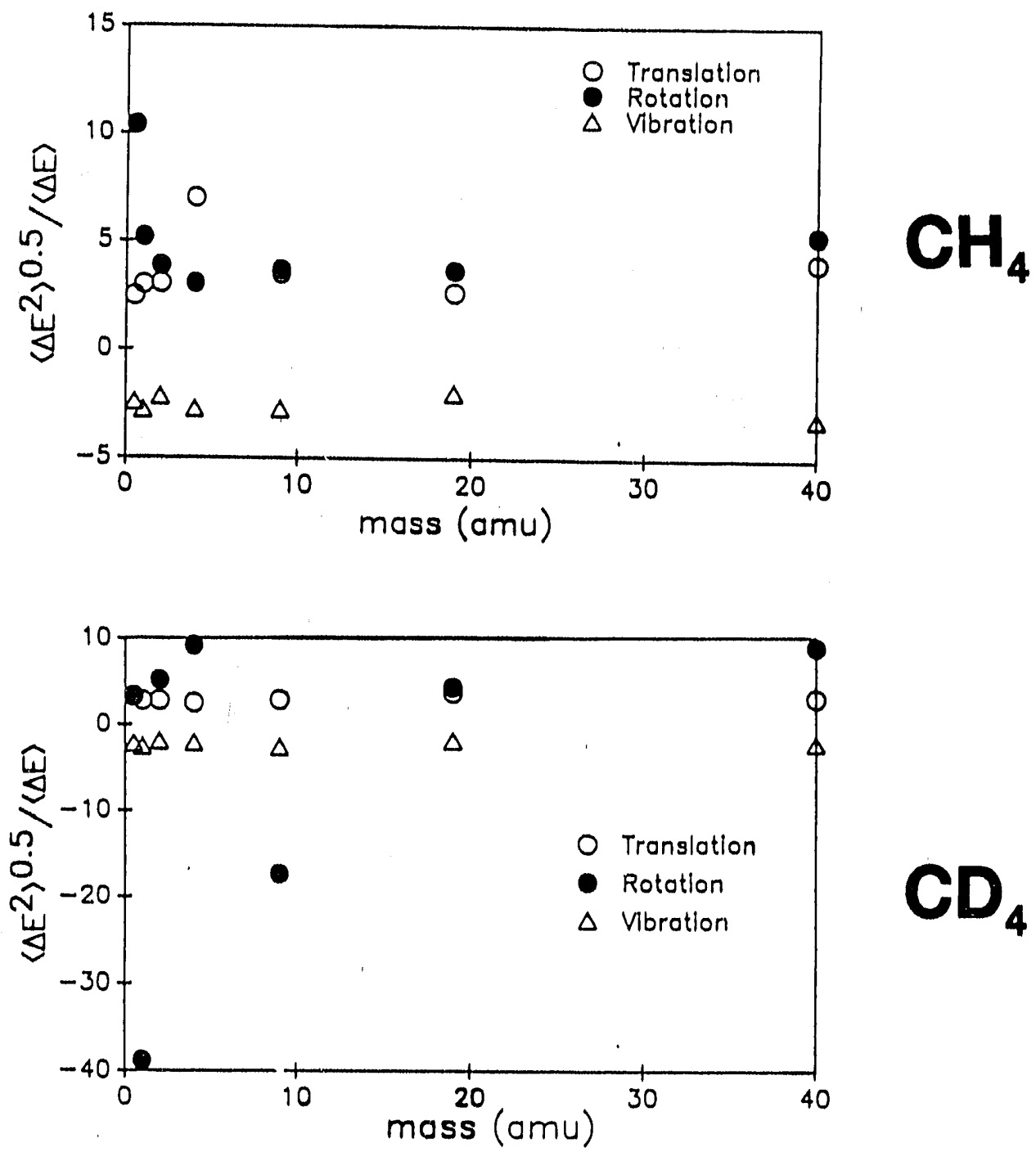

$\mathrm{CD}_{4}$

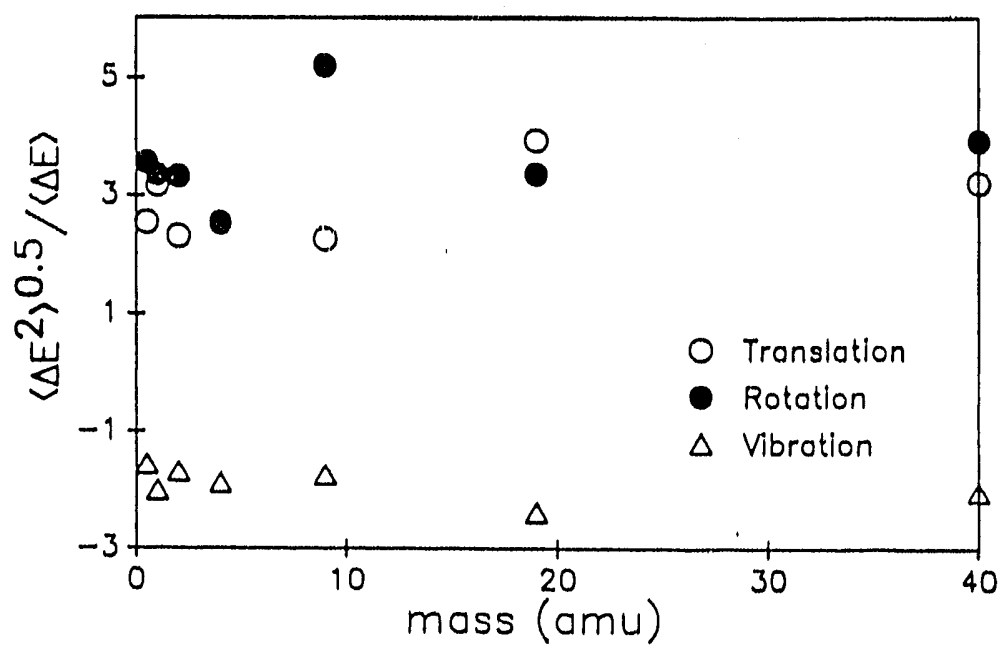

$\mathrm{CF}_{4}$

Fig. 13. Plots of $\left\langle\Delta E^{2}\right\rangle_{\text {all }}^{0.5} /\langle\Delta E\rangle_{\text {all }}$ vs mass of deactivator for $\mathrm{CH}_{4}, \mathrm{CD}_{4}$ and $C F_{4}$ systems. 


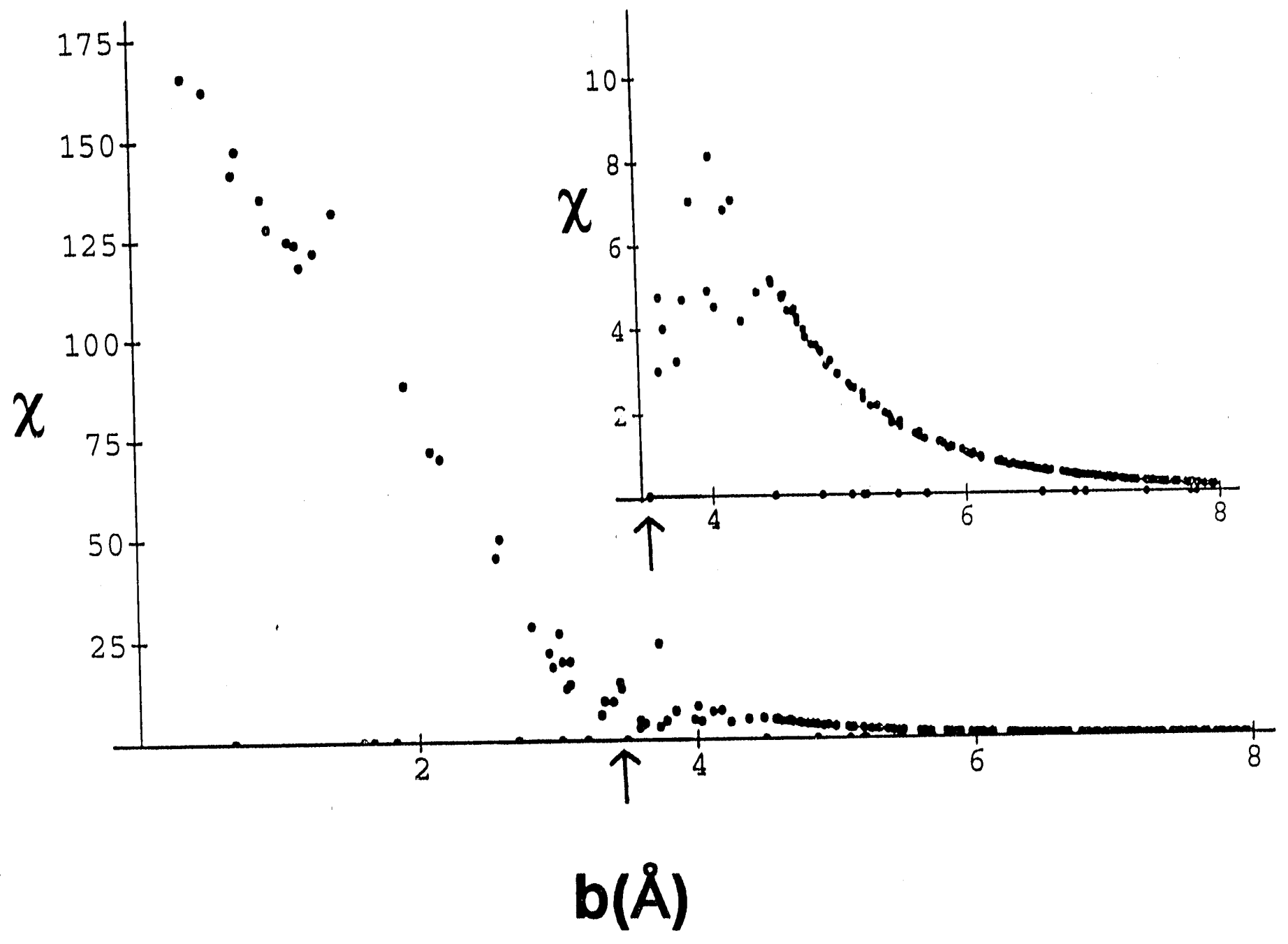

Fig 14. The scattering angle (degrees) vs impact parameter (A) for $\mathrm{CH}_{4}+$ mass 40 from 200 trajectories. The arrow indicates the hard sphere diameter. The inset illustrates that the interaction is important past the hard sphere diameter. 


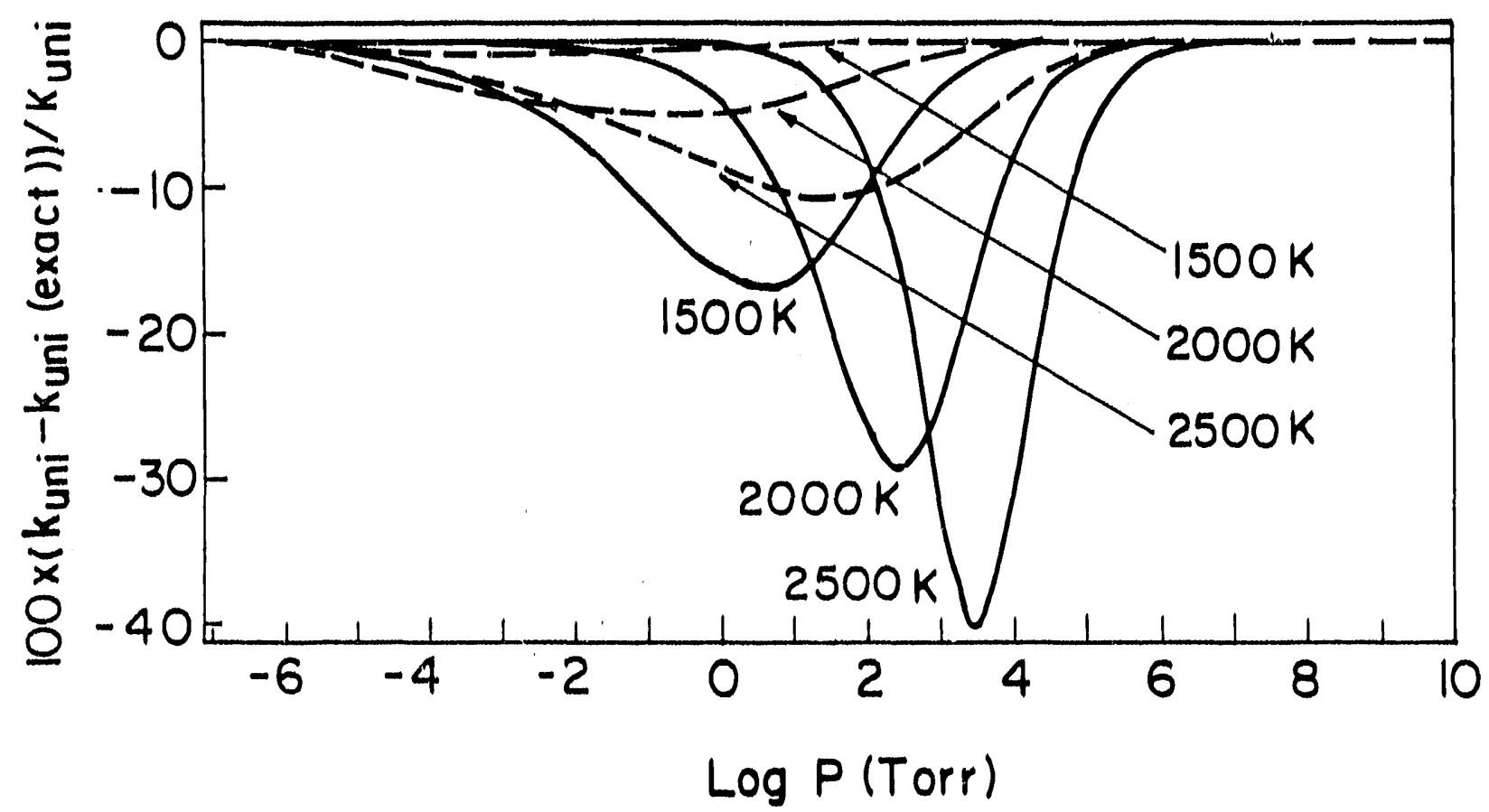

Fig. 15. Percent deviation of the exact rate coefficient from the approximate one vs logarithm of the pressure for quadricyclane isomerization.

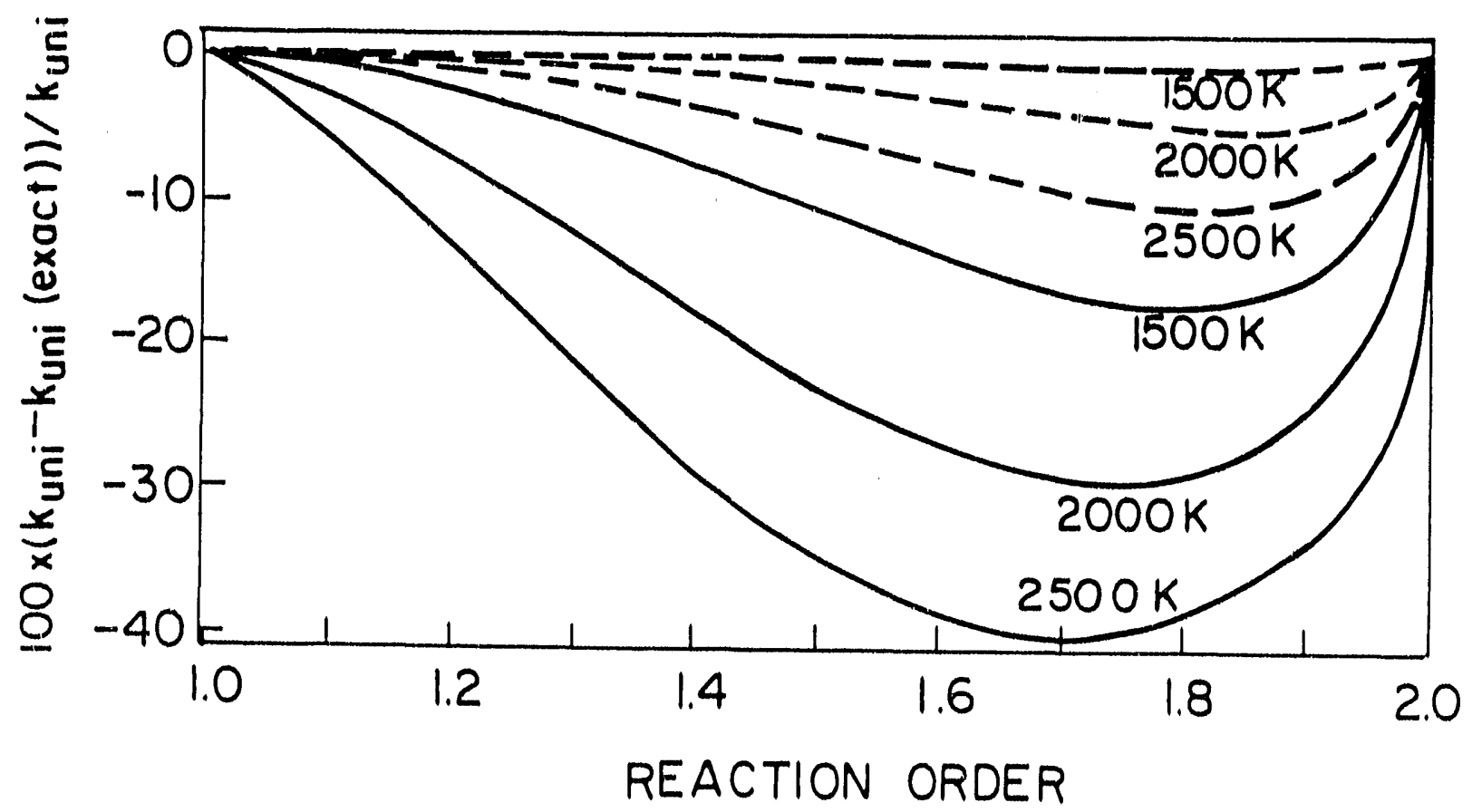

Fig. 16. Percent deviation of the exact rate coefficient from the approximate rate coefficient vs the reaction order for quadricyclane isomerization. 


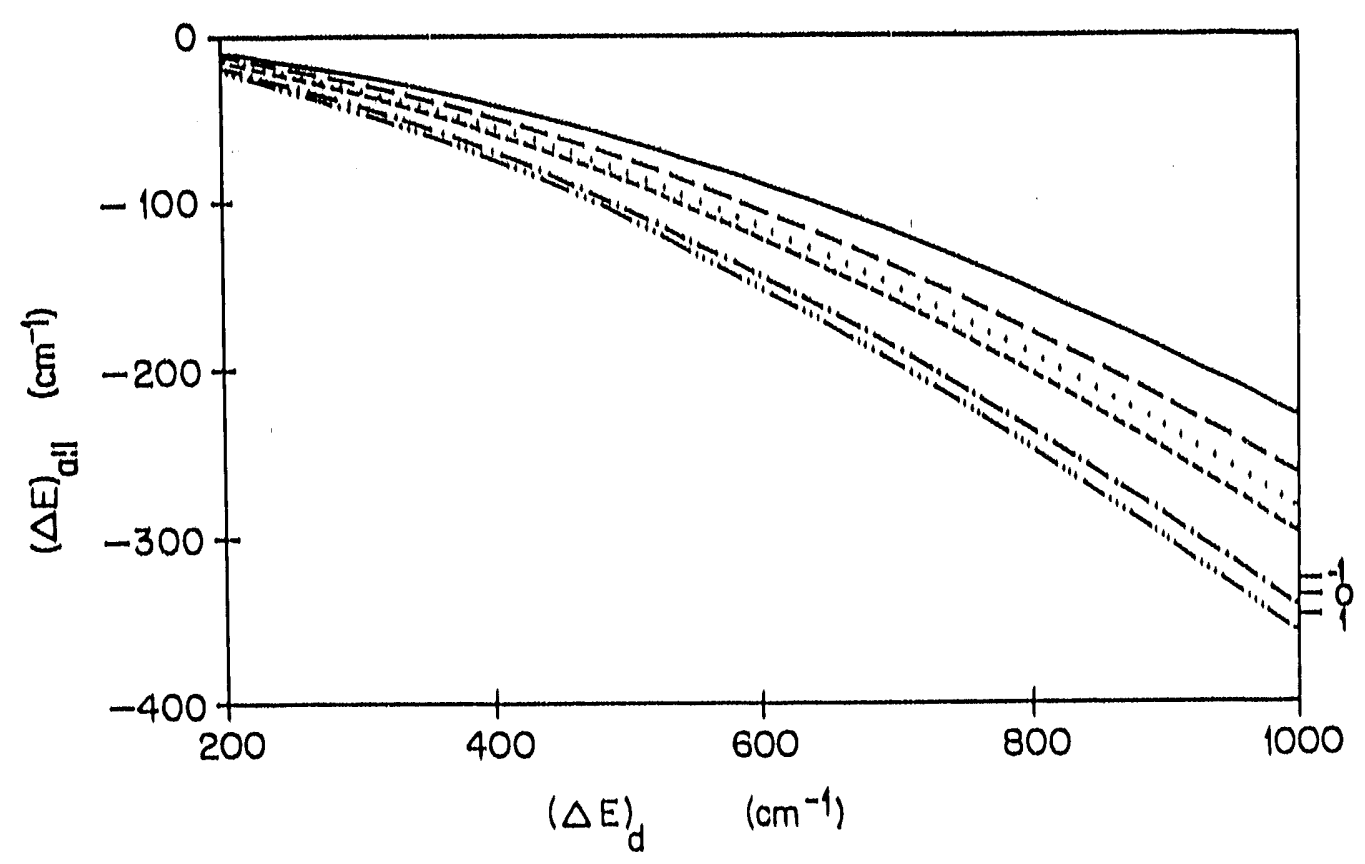

Fig. 17. Plots of $\langle\Delta E\rangle_{\text {all }} v s\langle\Delta E\rangle_{\text {down }}$ for the cyclohexene system at $1200 \mathrm{~K}$ for eq 9 (short dotted line) and eq 10 for energies (kcal/mole) of $61.9=E_{0}$ (solid line), $69.8=\left\langle E_{r, 0}\right.$ (dashed line), 80 (short dashed line), 100m $\langle E\rangle_{r, \text { xppt }}$ (dash-dot line), and $108.6=\langle E\rangle_{r, 0}$ (dash-dot-dot-dot line). Indicial marks for $\langle\Delta E\rangle_{\text {all }}$ from eq 10 are placed for $\langle\Delta E\rangle_{\text {down }}=$ $1000 \mathrm{~cm}^{-1}$ with $\langle E\rangle_{\text {, }}(\mathrm{kcal} / \mathrm{mole})$ of 92.6 for $p=0.1$ Torr (designated -1$), 98.1$ for $p=1$ Torr (designated 0 ), and 103.2 for $p=10$ Torr (designated 1 ).

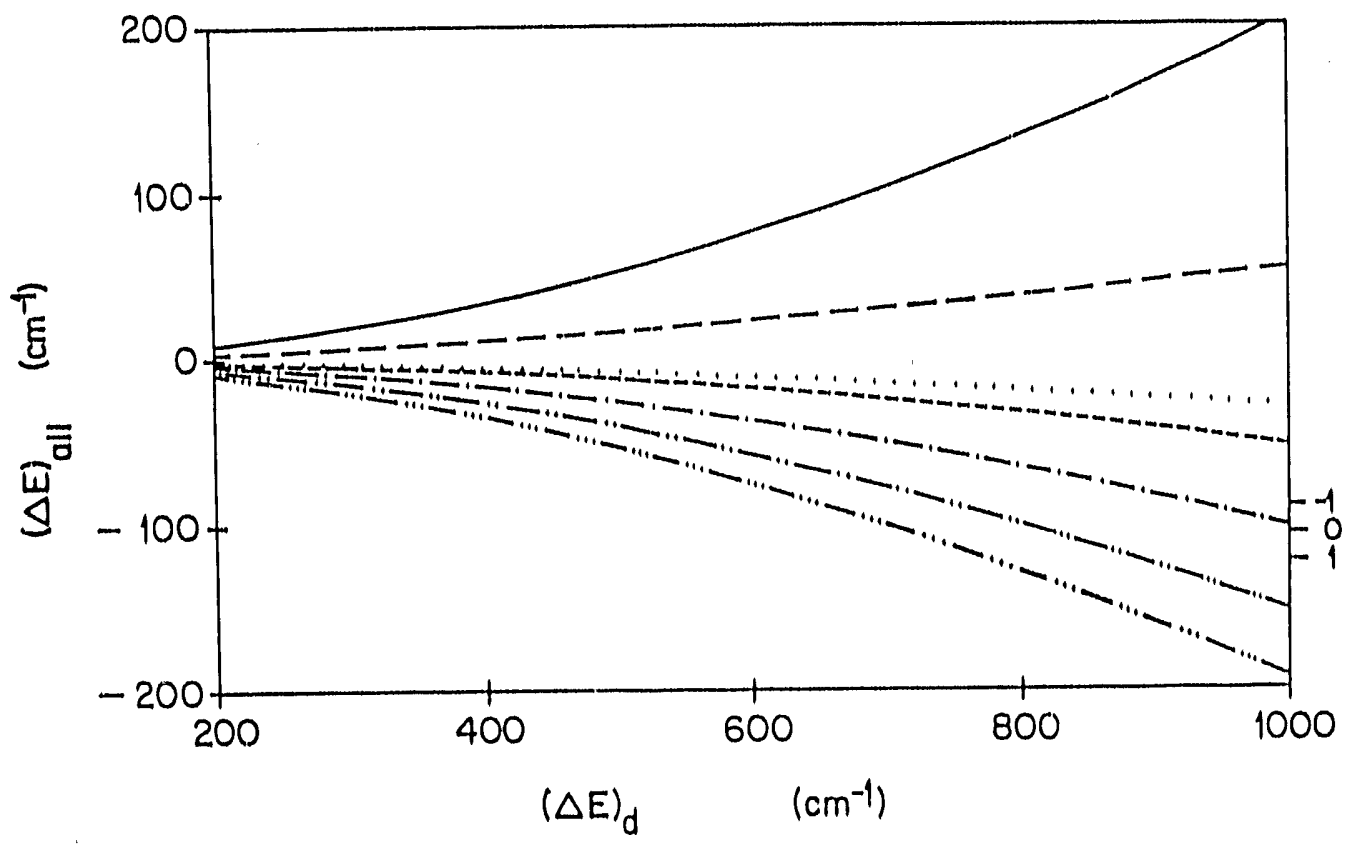

Fig. 18. Plots of $\langle\Delta E\rangle_{\text {all }} v s\langle\Delta E\rangle_{\text {down }}$ for the cyclohexene system at $2000 \mathrm{~K}$ for eq 9 (short dotted line) and eq 10 for energies (kcal $/ \mathrm{mole}$ ) of $61.9=E_{0}$ (solid line), $80=\langle E\rangle_{r .0}$ (dashed line), $102.6=\langle E\rangle_{1,0}$ (short dashed line), 118= $\langle E\rangle_{\text {rexpt }}$ (dash-dot line), 140 (dashdot-dot line), and $165.5=\langle E\rangle_{\text {r.o }}$ (dash-dot-dot-dot line). Indicial marks for $\langle\Delta E\rangle_{\text {all }}$ from eq 10 are placed for $\langle\Delta E\rangle_{\text {down }}=1000 \mathrm{~cm}^{-1}$ with $\langle E\rangle_{\text {r,p }}$ ( $(\mathrm{kcal} / \mathrm{mole}$ ) of 115.1 for $p=0.1$ Torr (designated -1 ), 120.9 for $p=1$ Torr (designated 0 ), and 128.8 for $p=10$ Torr (designated 1). 
Table is Hard Sphere Coillalon Rato Coetficlents $\left(10^{.10} \mathrm{co} / \mathrm{molec} \cdot \mathrm{sec}\right)$ [collision dlameter $\ln \boldsymbol{A} \ln ()]$

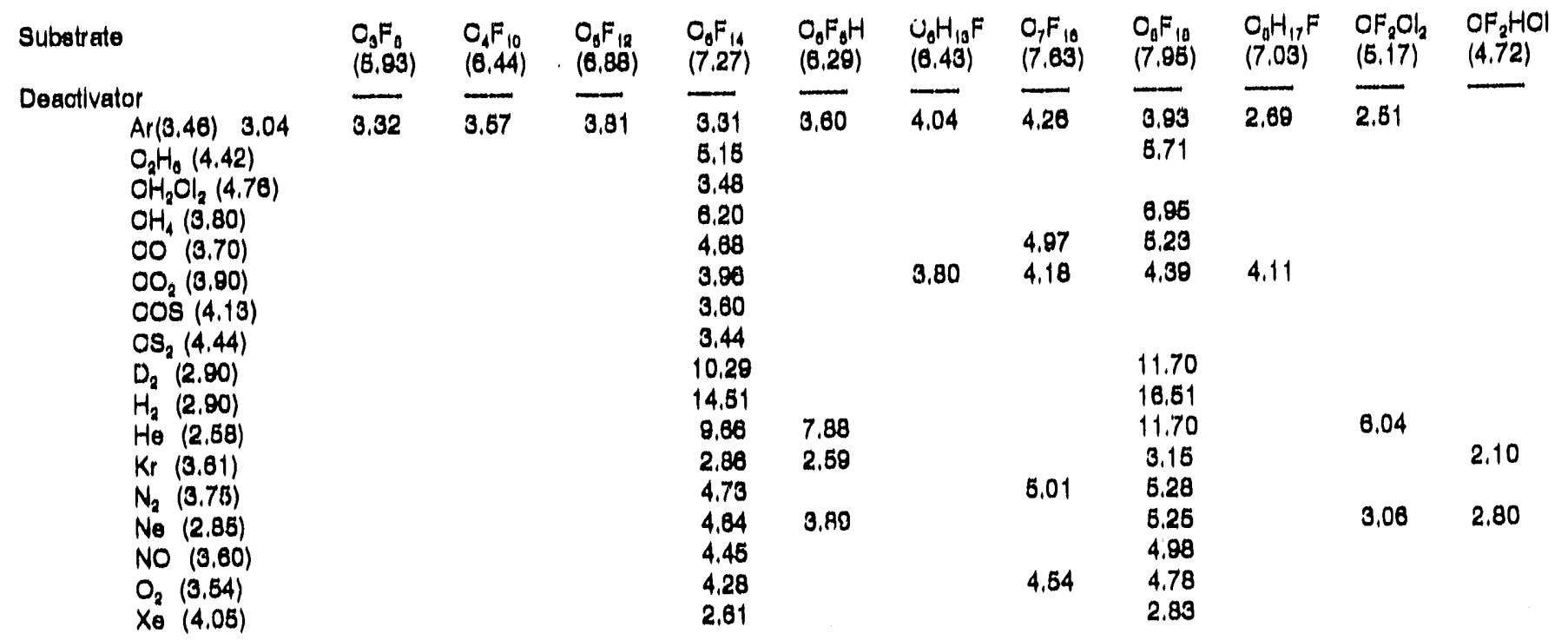

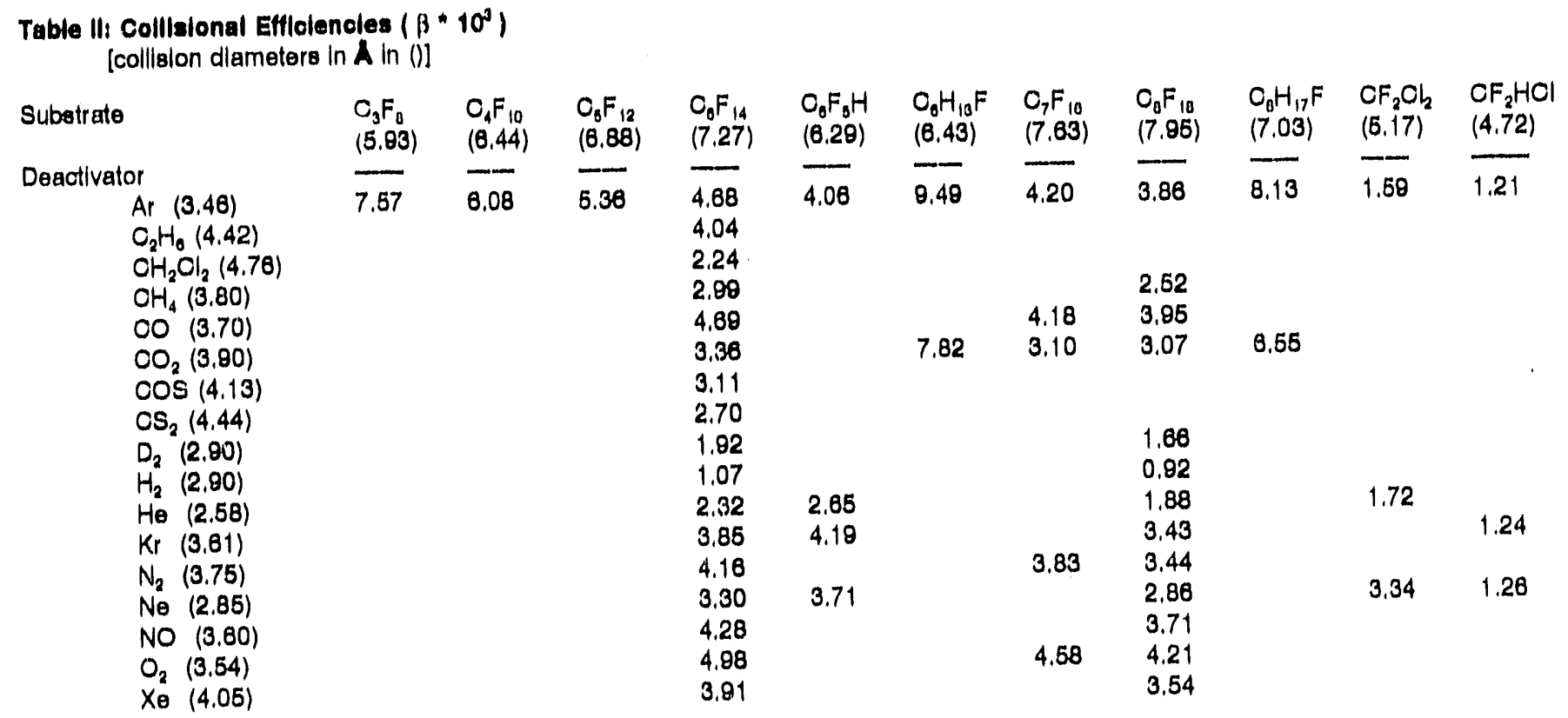


Table Illi 8ystem Parametere

Hard sphere dlameter: $3.47 \mathrm{~A}$ (caloulated)

Inltial Energles (kcal/mole):

$E_{w}=100$

$F_{11 x}=3.0$

$E_{t 1 \text { mant }}=2.6$

$\frac{\mathrm{CH}_{4}}{16}$
0.823
$3.17(3)$
$2069(9)$
10004
$6.610^{2}$
1.066

$C D_{4}$

\section{0}

1.714

$6.34(3)$

$1522(\theta)$

7363

$5.710^{3}$

1.068
$\mathrm{CF}_{1}$

\section{8}

7.356

$59.75(3)$

$633(8)$

3350

$7.010^{\circ}$

1.076 $\rho(35000) / \rho(34650)$

" reduced mass, "moment of Inertia. "vibration frequency, "zero point energy. " density of Internal energy states

- Geometrio Means:

$\begin{array}{lllll}\mathrm{CH}_{4} & 3184(3) & 3022(1) & 1683(2) & 1413(3) \\ O D_{4} & 2386(3) & 2141(3) & 1121(1) & 1062(3) \\ O F_{4} & 1338(3) & 686(3) & 410(3) & 365(2)\end{array}$

Table IV: Average Energy Transferred (kcal/mole)

mass of Deactivator (amu)

$40 \quad 1809-4$

2

0.460

0.790

0.513

0.807

0.414
0.707

1.151

$0.46 \theta$

$-0.087$

0.351

0.486

0.273

$-0.852$

$-0.754$

$-1.602$
$-0.763$

$-0,688$

$-1.476$

$$
\begin{aligned}
& 0.241 \\
& 1.067
\end{aligned}
$$

1.362

0.817

0.841

0.868

0.508

0,181

0,558

0.369

0.305

0.342

$-0.607$

$-1.267$

$-2.036$
$-1.080$

$-0.863$

$-1.368$
1.132

1.150

0.588

0.287

$-0.034$

0.318

$-1.266$

$-1.155$

$-0.880$
0.5

1.532

0.822

0.470

0.132

0.425

0.282

$-1.481$

$-1.356$

$-0.828$ 
Table V: Average Energy Quanttles (kcal/mole) for Strong Collider

\begin{tabular}{|c|c|c|c|c|c|c|c|c|}
\hline $\begin{array}{l}T, K \\
1200 \\
1500 \\
1800 \\
2000\end{array}$ & $\begin{array}{l}\langle E\rangle_{\text {wal }} \\
42.6 \\
62.5 \\
83.7 \\
88.4\end{array}$ & $\begin{array}{l}\infty \text { Torr } \\
108.6 \\
128.8 \\
150.5 \\
165.5\end{array}$ & $\begin{array}{l}10 \text { Torr } \\
103.2 \\
113.2 \\
122.2 \\
128.8\end{array}$ & $\begin{array}{c}1 \text { Torr } \\
98.1 \\
105.8 \\
114.1 \\
120.8\end{array}$ & $\begin{array}{c}0.1 \text { Torr } \\
92.6 \\
99.2 \\
107.6 \\
115.1\end{array}$ & $\begin{array}{c}0 \text { Torr } \\
69.8 \\
77.4 \\
90.6 \\
102.6\end{array}$ & $\begin{array}{c}E_{\text {XN }}{ }^{\circ} \\
\sim 78 \\
80 \\
\sim 80 \\
\sim 98\end{array}$ & $\begin{array}{l}\left\langle E_{1,0 \times p}{ }^{d}\right. \\
100 \\
105 \\
113 \\
118\end{array}$ \\
\hline
\end{tabular}

"Oalculated from JEf(E)dE where $f(E)$ is the normalized Boltzmann distribution. "Oaloulated from $E_{0}+\int E G(E) d E$ where $G(E)=[k(E) /(\omega+k(E))] f(E) / \int[k(E) /(\omega+k(E))] f(E) d E .{ }^{\circ} E_{a q}$ is the approximate energy which causes the $\langle\Delta E\rangle$ ve $\langle\Delta E\rangle_{\text {dam }}$ curve from eq 10 to overlap the curve calculated from eq $\theta$. "Mean average energy of reacting molecules from experimental data of $\mathrm{KS}$ obtained by averaging values of $\langle E\rangle_{1,0}$ determined from $k / K_{m}$ and $E_{a p}$ at 350 and 150 Torr of krypton.

Table V: Dependence of $<\Delta E>$ Quanttles on Temperature

\begin{tabular}{|c|c|c|c|c|}
\hline \multirow[b]{2}{*}{$T, K$} & \multicolumn{2}{|c|}{ 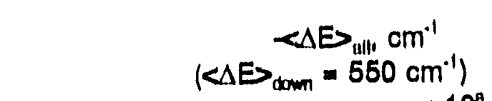 } & \multicolumn{2}{|c|}{$\begin{array}{c}<\Delta E>_{\mathrm{dimm}} \mathrm{cm}^{-1} \\
\left(-\Delta E>_{\mathrm{wll}}=73 \mathrm{~cm}^{-1}\right)\end{array}$} \\
\hline & $\theta q 9$ & eq $10^{\mathrm{A}}$ & $\theta q \theta$ & $\operatorname{\theta q} 10^{\mathrm{a}}$ \\
\hline $\begin{array}{l}1200 \\
1500 \\
1800 \\
2000\end{array}$ & $\begin{array}{c}97 \\
49 \\
20 \\
9^{b}\end{array}$ & $\begin{array}{c}120 \\
78 \\
47 \\
31\end{array}$ & $\begin{array}{c}470 \\
680 \\
1080 \\
1600\end{array}$ & $\begin{array}{l}420 \\
530 \\
680 \\
850\end{array}$ \\
\hline
\end{tabular}

acalculated at the values of $\langle E\rangle$, given in Table $V$. "Values not yet negative since relevant energy has risen to $\sim 118 \mathrm{kcal} / \mathrm{mole}$ (8ea Table $\mathrm{V}$ ); inversion energy at $2000 \mathrm{~K}$ is estimated as $\sim 80 \mathrm{kcal} / \mathrm{mole}$. 

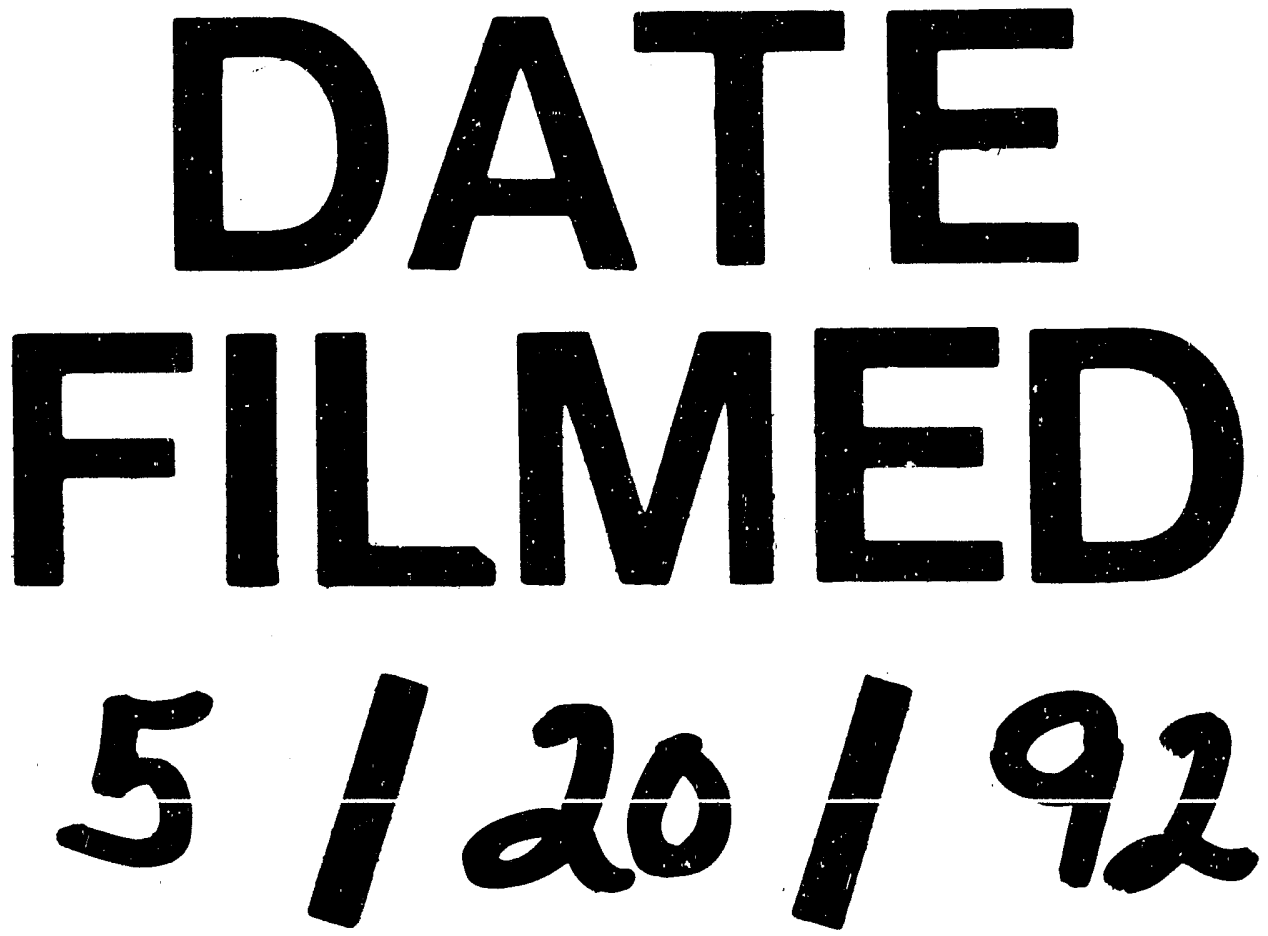
(2) 